\title{
Catalytic Asymmetric Rearrangement of Allylic Trichloroacetimidates. A Practical Method for Preparing Allylic Amines and Congeners of High Enantiomeric Purity
}

\section{Carolyn E. Anderson and Larry E. Overman*}

\section{Supporting Information}

Representative experimental procedures, copies of HPLC and GC traces used to determine enantiopurity, and copies of ${ }^{1} \mathrm{H}$ and ${ }^{13} \mathrm{C}$ NMR spectra for new compounds.

(38 Pages)

General experimental details: The procedure employed to purify $\mathrm{THF}$ and $\mathrm{CH}_{2} \mathrm{Cl}_{2}$ has been described; ${ }^{1}$ other general experimental details also have been described. ${ }^{2}$ All HPLC and GC analyses used to determine enantiomeric purity were calibrated with samples of the racemate.

General procedure for the synthesis of trichloracetimidates. Preparation of $(E)$-2-


hexen-6-oic acid trichloroacetimidate methyl ester $(1 \mathrm{k})$. Trichloroacetonitrile $(0.36 \mathrm{~mL}, 3.6 \mathrm{mmol})$ was added to 6 hydroxyhex-4-enoic acid methyl ester $^{3}$ (310 $\left.\mathrm{mg}, 2.4 \mathrm{mmol}\right)$, DBU (70 $\square \mathrm{L}, 0.48 \mathrm{mmol})$, and $\mathrm{CH}_{2} \mathrm{Cl}_{2}(15 \mathrm{~mL})$ at $0{ }^{\circ} \mathrm{C}$. The reaction was warmed slowly to room temperature. After $2 \mathrm{~h}$, the reaction was concentrated. Purification of the residue by chromatography (Davisil grade $\mathrm{SiO}_{2}$, 95:5 hexanes/EtOAc) afforded $622 \mathrm{mg}(95 \%)$ of $\mathbf{1 k}$ as a colorless oil: ${ }^{1} \mathrm{H}$ NMR $(500 \mathrm{MHz}$, $\left.\mathrm{CDCl}_{3}\right) \square 8.28$ (broad s, $\left.1 \mathrm{H}\right), 5.83-5.92(\mathrm{~m}, 1 \mathrm{H}), 5.70-5.77(\mathrm{~m}, 1 \mathrm{H}), 4.74(\mathrm{~d}, J=6.1 \mathrm{~Hz}$, 2H), 3.67 (s, 3H), 2.38-2.47 (m, 4H); $\left.{ }^{13} \mathrm{C} \mathrm{NMR} \mathrm{(125} \mathrm{MHz,} \mathrm{CDCl}_{3}\right) \square 173.1,162.4,134.2$, 124.3, 91.4, 69.4, 51.5, 33.2, 27.4; IR (neat) 3344, 2958, 1738, 1661, 1444, $1290 \mathrm{~cm}^{-1}$; HRMS $\left(\mathrm{CI}-\mathrm{NH}_{3}\right) \mathrm{m} / \mathrm{z} 286.9881$ [286.9883 calcd for $\mathrm{C}_{9} \mathrm{H}_{12} \mathrm{Cl}_{3} \mathrm{NO}_{3}(\mathrm{M})$ ]. Anal. Calcd for $\mathrm{C}_{9} \mathrm{H}_{12} \mathrm{Cl}_{3} \mathrm{NO}_{3}$ : C, 37.46; H, 4.19; N, 4.85. Found: C, 37.61; H, 4.18; N, 4.90.

\footnotetext{
${ }^{1}$ Pangborn, A. B.; Giardello, M. A.; Grubbs, R. H.; Rosen, R. K.; Timmers, F. J. Organometallics, 1996, $15,1518$.

${ }^{2}$ Deng, W.; Overman, L. E. J. Am. Chem. Soc. 1994, 116, 11241.

${ }^{3}$ Analogous to 6-hydroxyhex-4-enoic acid ethyl ester: Casara, P. Tetrahedron Lett. 1994, 35, 3049-3050.
} 
General procedure for allylic imidate rearrangement using 5 mol \% COP-Cl (5).

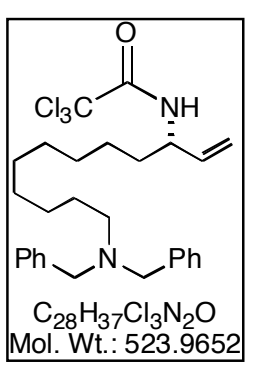

Preparation of $(S)-2,2,2-t$ richloro- $N-($ (9-( $N, N$-dibenyl)-1nonanylaminoallyl)acetamide (2q). A solution of $\mathrm{COP}-\mathrm{Cl}(\mathbf{5}, 12 \mathrm{mg}$, $0.008 \mathrm{mmol})$ in $\mathrm{CH}_{2} \mathrm{Cl}_{2}(0.26 \mathrm{~mL})$ was added to imidate $1 \mathbf{q}(81 \mathrm{mg}, 0.16$ mmol). The reaction flask was then sealed, protected from light and maintained at room temperature. After $18 \mathrm{~h}$, the reaction was concentrated. Purification of the residue by chromatography (Davisil grade $\mathrm{SiO}_{2}, 98: 2$ hexanes/EtOAc) afforded $67 \mathrm{mg}(82 \%)$ of $\mathbf{2 q}$ as a pale yellow oil. Chiral HPLC (Chiracel OD-H, 99.6:0.4 $n$-hexane/IPA, 0.8 mL/min, $230 \mathrm{~nm}$ ) showed that $2 \mathbf{q}$ had been formed in $97 \%$ ee: $[\square]^{27}{ }_{D}=+7.1,[\square]^{27}{ }_{577}=+6.2$, $[\square]^{27}{ }_{546}=+7.1$, $[\square]_{435}^{27}=+6.9,[\square]^{27}{ }_{405}=+10.2\left(\mathrm{c} 0.29, \mathrm{CHCl}_{3}\right) ;{ }^{1} \mathrm{H}$ NMR $\left(500 \mathrm{MHz}, \mathrm{CDCl}_{3}\right) \square 7.19-7.39$ $(\mathrm{m}, 10 \mathrm{H}), 6.50(\mathrm{~d}, J=8.1 \mathrm{~Hz}, 1 \mathrm{H}), 5.79(\mathrm{ddd}, J=17.0,10.4,5.6 \mathrm{~Hz}, 1 \mathrm{H}), 5.23$ (d, $J=$ $17.2 \mathrm{~Hz}, 1 \mathrm{H}), 5.19(\mathrm{~d}, J=10.5 \mathrm{~Hz}, 1 \mathrm{H}), 4.37-4.44(\mathrm{~m}, 1 \mathrm{H}), 3.54(\mathrm{~s}, 4 \mathrm{H}), 2.39$ (t, $J=6.9$ $\mathrm{Hz}, 2 \mathrm{H}) 1.45-1.71(\mathrm{~m}, 4 \mathrm{H}), 1.15-1.40(\mathrm{~m}, 12 \mathrm{H}) ;{ }^{13} \mathrm{C} \mathrm{NMR}\left(125 \mathrm{MHz}, \mathrm{CDCl}_{3}\right) \square 161.1$, $139.8,136.7,129.7,128.8,128.1,126.8,116.0,92.8,58.2,53.5,53.3,34.4,29.41,29.35$, 29.18, 29.15, 26.8, 25.5; IR (neat) 3329, 3028, 2927, 2858, 2796, 1699, 1514, 1452, 1244 $\mathrm{cm}^{-1}$; HRMS $\left(\mathrm{CI}-\mathrm{NH}_{3}\right) \mathrm{m} / z$ 523.2039 [523.2050 calcd for $\mathrm{C}_{28} \mathrm{H}_{38} \mathrm{Cl}_{3} \mathrm{~N}_{2} \mathrm{O}(\mathrm{M}+\mathrm{H})$ ]. Anal. Calcd for $\mathrm{C}_{28} \mathrm{H}_{37} \mathrm{Cl}_{3} \mathrm{~N}_{2} \mathrm{O}$ : C, 64.18; H, 7.12; N, 5.35. Found: C, 63.89; H, 7.06; N, 5.28.

General procedure for allylic imidate rearrangement using $5 \mathrm{~mol} \% \mathrm{COP}-\mathrm{Cl}(5)$ at Mol. Wt.: 284.6098 $38{ }^{\circ} \mathrm{C}$. Preparation of $(\mathrm{S})-2,2,2-$ trichloro- $\mathrm{N}-(1-$ cyclohexylallyl)acetamide (2f). A solution of $\mathrm{COP}-\mathrm{Cl}(\mathbf{5}, 129 \mathrm{mg}$, $0.088 \mathrm{mmol})$ in $\mathrm{CH}_{2} \mathrm{Cl}_{2}(1.76 \mathrm{~mL})$ was added to imidate $\mathbf{1 f}(500 \mathrm{mg}$, $1.76 \mathrm{mmol})$. The reaction flask was then sealed, protected from light and maintained at $38{ }^{\circ} \mathrm{C}$. After $18 \mathrm{~h}$, the reaction was concentrated. Purification of the residue by chromatography (Davisil grade $\mathrm{SiO}_{2}$, 99.5:0.5 hexanes/EtOAc) afforded $412 \mathrm{mg}(82 \%)$ of $\mathbf{2 f}$ as a colorless solid. Chiral HPLC (Chiracel OD-H, 99:1 $n$-hexane/IPA, $0.8 \mathrm{~mL} / \mathrm{min}, 230 \mathrm{~nm}$ ) showed that $2 \mathrm{f}$ had been


$[\square]_{435}^{27}=+60.1,[\square]^{27}{ }_{405}=+71.4\left(\mathrm{c}_{0.42}, \mathrm{CHCl}_{3}\right) ;{ }^{1} \mathrm{H} \mathrm{NMR}\left(500 \mathrm{MHz}, \mathrm{CDCl}_{3}\right) \square 6.58$ (broad s, 1H), 5.79 (ddd, $J=17.1,10.5,6.0 \mathrm{~Hz}, 1 \mathrm{H}), 5.19-5.25(\mathrm{~m}, 2 \mathrm{H}), 4.27$ (dd, $J=$ $14.8,6.2 \mathrm{~Hz}, 1 \mathrm{H}), 1.65-1.81(\mathrm{~m}, 5 \mathrm{H}), 1.51-1.60(\mathrm{~m}, 1 \mathrm{H}), 0.95-1.30(\mathrm{~m}, 5 \mathrm{H}) ;{ }^{13} \mathrm{C} \mathrm{NMR}$ $\left(125 \mathrm{MHz}, \mathrm{CDCl}_{3}\right) \square 161.2,135.3,116.7$, 93.0, 58.3, 41.9, 29.4, 28.5, 26.2, 25.9; IR (neat) 3298, 2927, 2858, 1692, 1522, 1452, $1251 \mathrm{~cm}^{-1}$; HRMS $\left(\mathrm{CI}-\mathrm{NH}_{3}\right) \mathrm{m} / z 284.0370$ [284.0376 calcd for $\mathrm{C}_{11} \mathrm{H}_{17} \mathrm{Cl}_{3} \mathrm{NO}(\mathrm{M}+\mathrm{H})$ ]. Anal. Calcd for $\mathrm{C}_{11} \mathrm{H}_{16} \mathrm{Cl}_{3} \mathrm{NO}$ : C, 46.42; $\mathrm{H}$, 5.67; N, 4.92. Found: C, 46.51; H, 5.66; N, 5.02. 
General procedure for allylic imidate rearrangement using 1 mol \% COP-Cl (5).

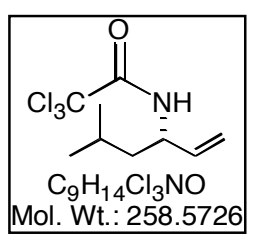

Preparation of $(S)$-2,2,2-Trichloro- $\mathrm{N}$-(1-iso-butylallyl)acetamide (2c).

A solution of $\mathrm{COP}-\mathrm{Cl}\left(\mathbf{5}, 0.13,0.12 \mathrm{M}\right.$ in $\left.\mathrm{CH}_{2} \mathrm{Cl}_{2}\right)$ was added to imidate

1c $(41 \mathrm{mg}, 0.16 \mathrm{mmol})$. The reaction flask was then sealed, protected from light and maintained at $38^{\circ} \mathrm{C}$. After $18 \mathrm{~h}$, the reaction was cooled to room temperature. Purification of the residue directly by chromatography (Davisil grade $\mathrm{SiO}_{2}, 99.5: 0.5$ hexanes/EtOAc) afforded $38 \mathrm{mg}(92 \%)$ of $\mathbf{2 c}$ as a colorless solid. Chiral HPLC (Chiracel OD-H, 99.5:0.5 $n$-hexane/IPA, $0.8 \mathrm{~mL} / \mathrm{min}, 230 \mathrm{~nm}$ ) showed that $2 \mathbf{c}$ had been formed in $98 \%$ ee $(S)$ : $[\square]^{27}=+9.0,[\square]_{577}^{27}=+7.1,[\square]_{546}^{27}=+4.6,[\square]_{435}^{27}=$ $+8.8,[\square]^{27}{ }_{405}=+12.5\left(\mathrm{c} 0.22, \mathrm{CHCl}_{3}\right) ; \mathrm{mp}: 69-71{ }^{\circ} \mathrm{C} ;{ }^{1} \mathrm{H} \mathrm{NMR}\left(500 \mathrm{MHz}, \mathrm{CDCl}_{3}\right) \square 6.46$ (broad s, 1H), 5.79 (ddd, $J=16.2,10.4,5.7 \mathrm{~Hz}, 1 \mathrm{H}), 5.24(\mathrm{td}, J=17.2,0.9 \mathrm{~Hz}, 1 \mathrm{H}), 5.18$ (td, $J=10.4,0.9 \mathrm{~Hz}, 1 \mathrm{H}), 4.45-4.53(\mathrm{~m}, 1 \mathrm{H}), 1.64-1.73(\mathrm{~m}, 1 \mathrm{H}), 1.47-1.52(\mathrm{~m}, 2 \mathrm{H})$, $0.96(\mathrm{~d}, J=6.6 \mathrm{~Hz}, 6 \mathrm{H}) ;{ }^{13} \mathrm{C} \mathrm{NMR}\left(125 \mathrm{MHz}, \mathrm{CDCl}_{3}\right) \square 161.1,136.9,115.8,92.8,51.9$, 43.6, 24.8, 22.7, 22.2; IR (neat) 3306, 2958, 1692, 1522, 1468, $1259 \mathrm{~cm}^{-1}$; HRMS (CI$\left.\mathrm{NH}_{3}\right) \mathrm{m} / z 199.9443$ [199.9437 calcd for $\left.\mathrm{C}_{5} \mathrm{H}_{5} \mathrm{Cl}_{3} \mathrm{NO}\left(\mathrm{M}-{ }^{i} \mathrm{Bu}\right)\right]$. Anal. Calcd for $\mathrm{C}_{9} \mathrm{H}_{14} \mathrm{Cl}_{3} \mathrm{NO}$ : C, 41.81; H, 5.46; N, 5.42. Found: C, 41.99; H, 5.46; N, 5.39. 
IH spectrum

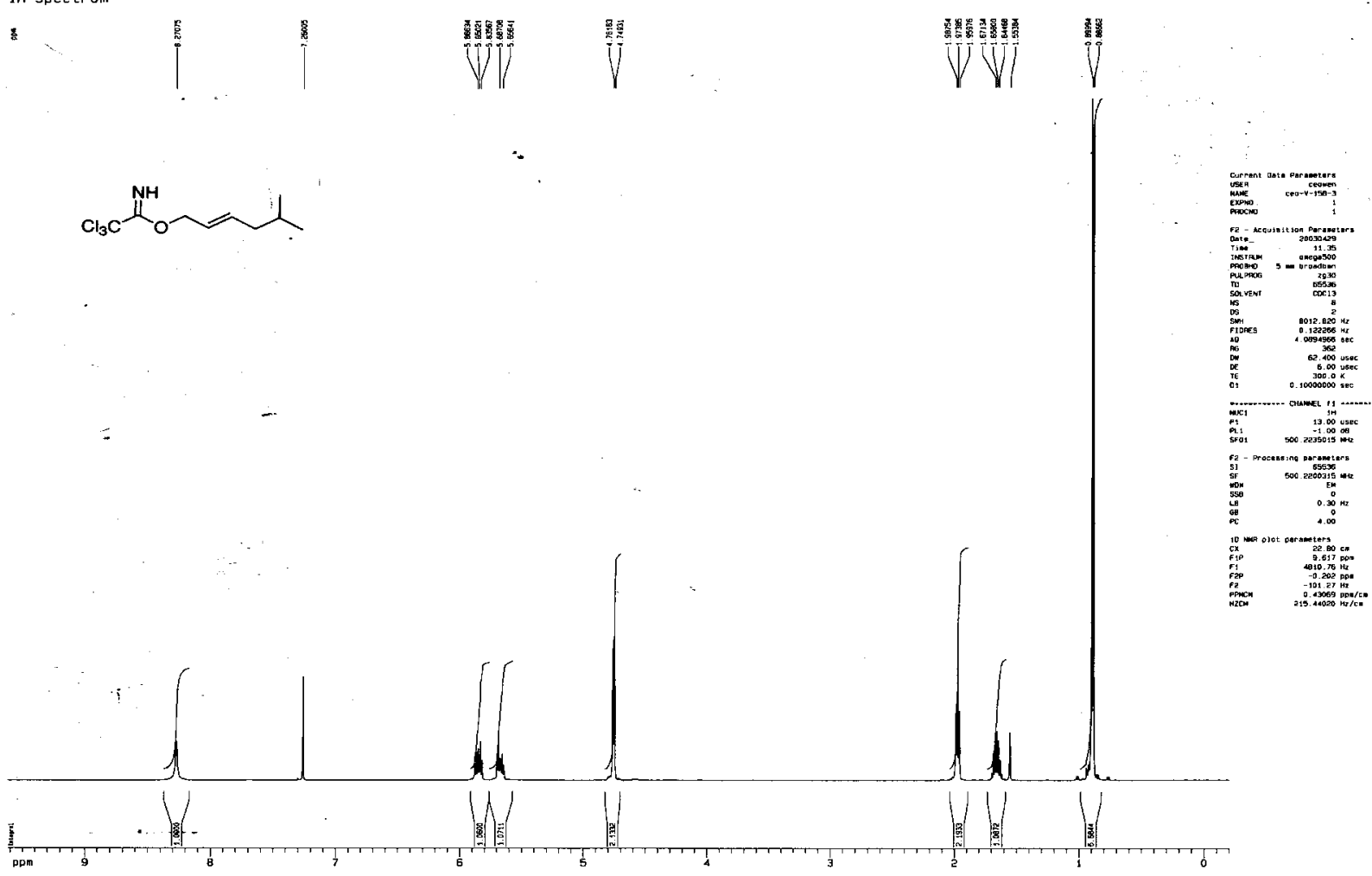

13C spectrum: with $1 \mathrm{H}$ decoupling

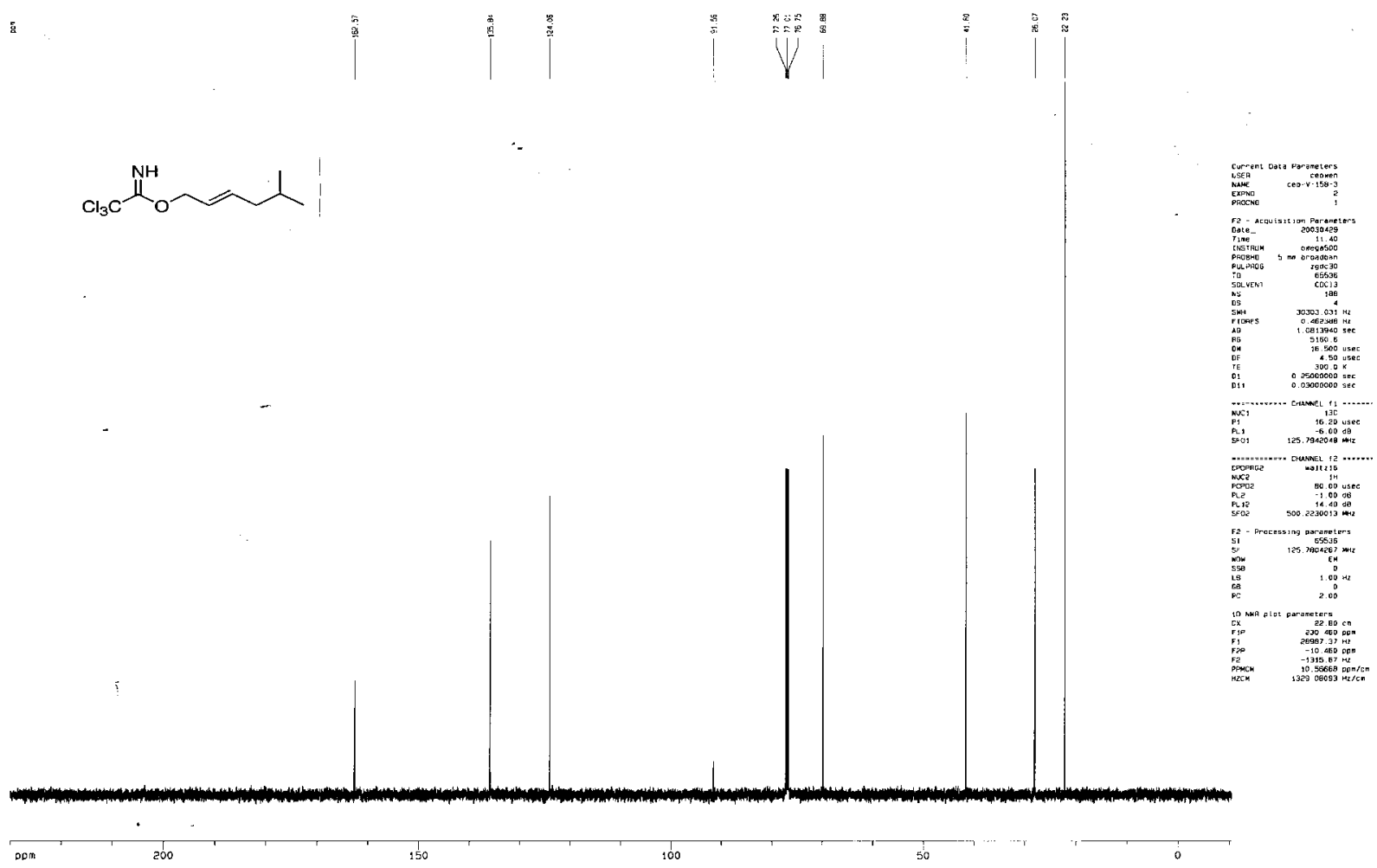


Supporting Information Anderson and Overman

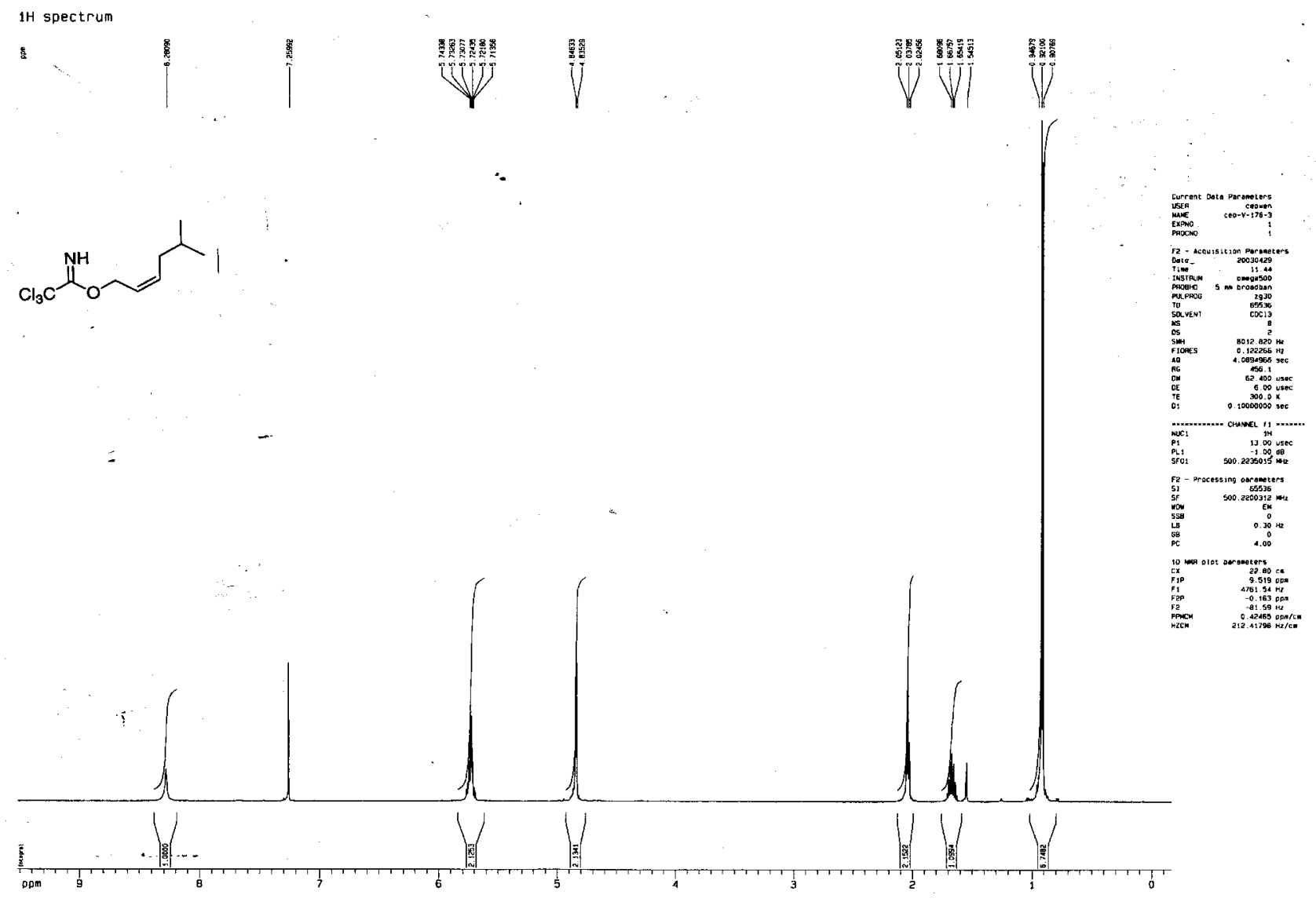

13 C spectrum with $1 \mathrm{H}$ decoupling




Supporting Information Anderson and Overman

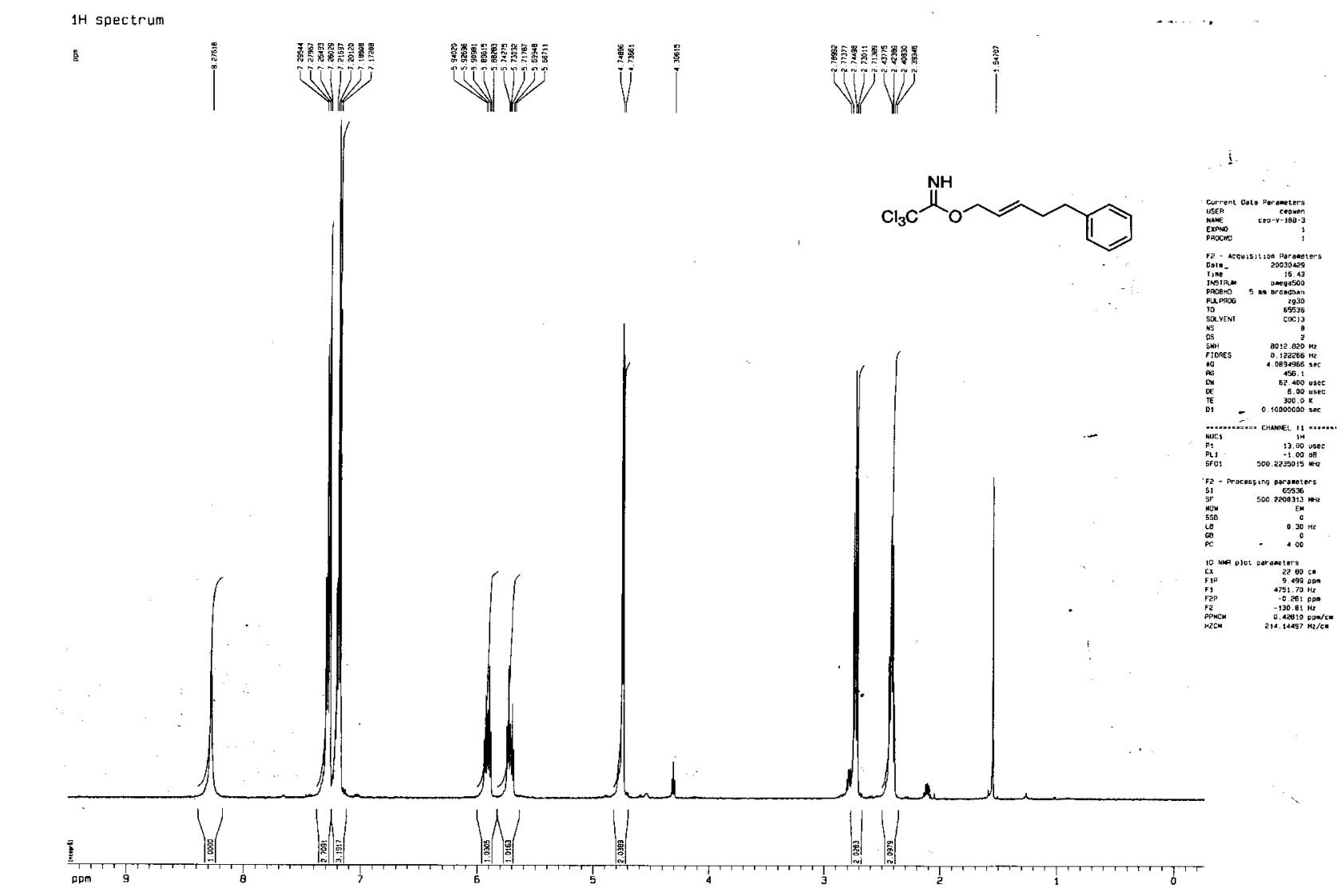

$13 \mathrm{C}$ spectrum with $1 \mathrm{H}$ decoupling

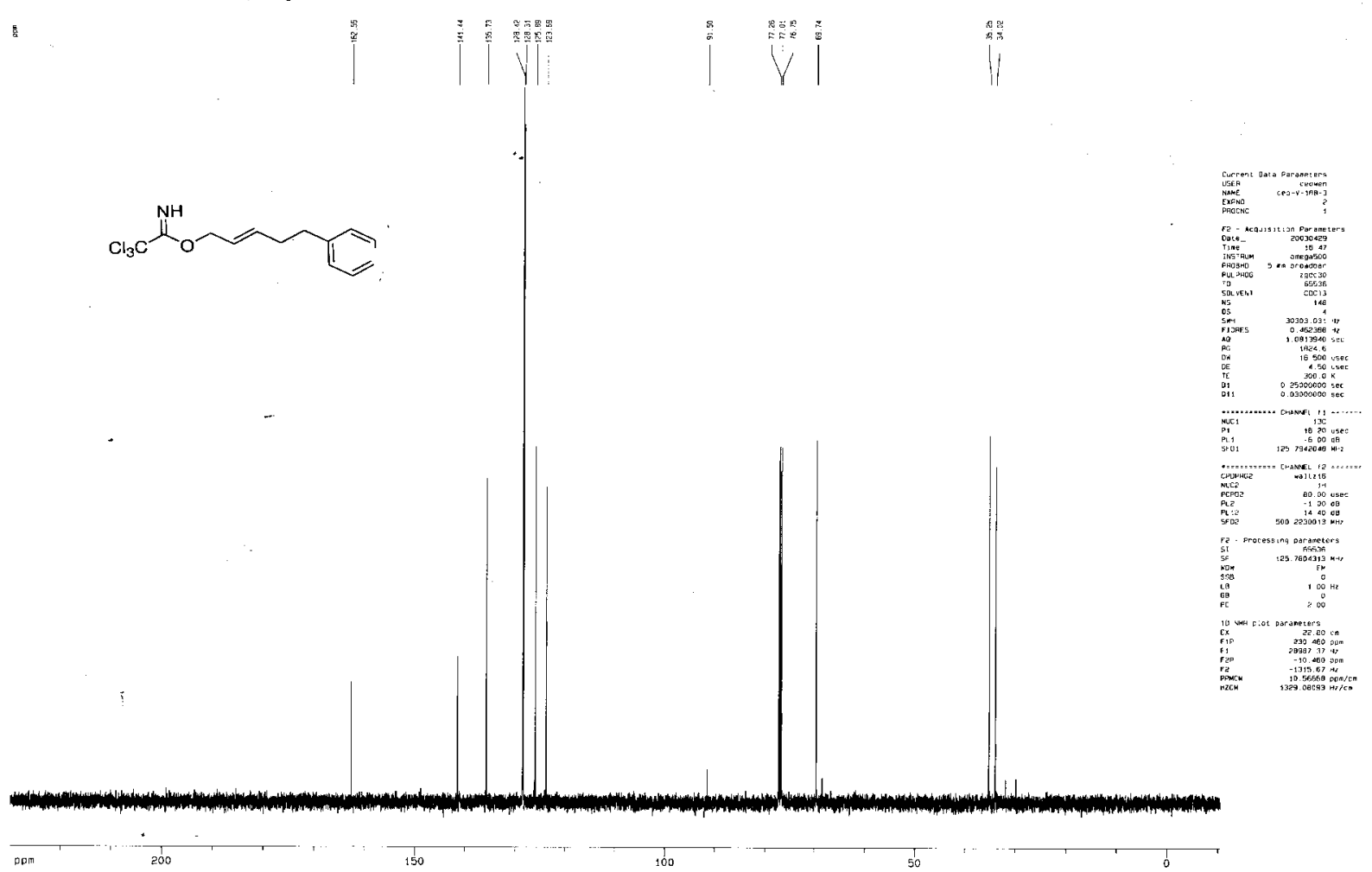


Supporting Information Anderson and Overman




Supporting Information Anderson and Overman




Supporting Information Anderson and Overman



13 Cpectrum with $1 \mathrm{H}$ decoupling

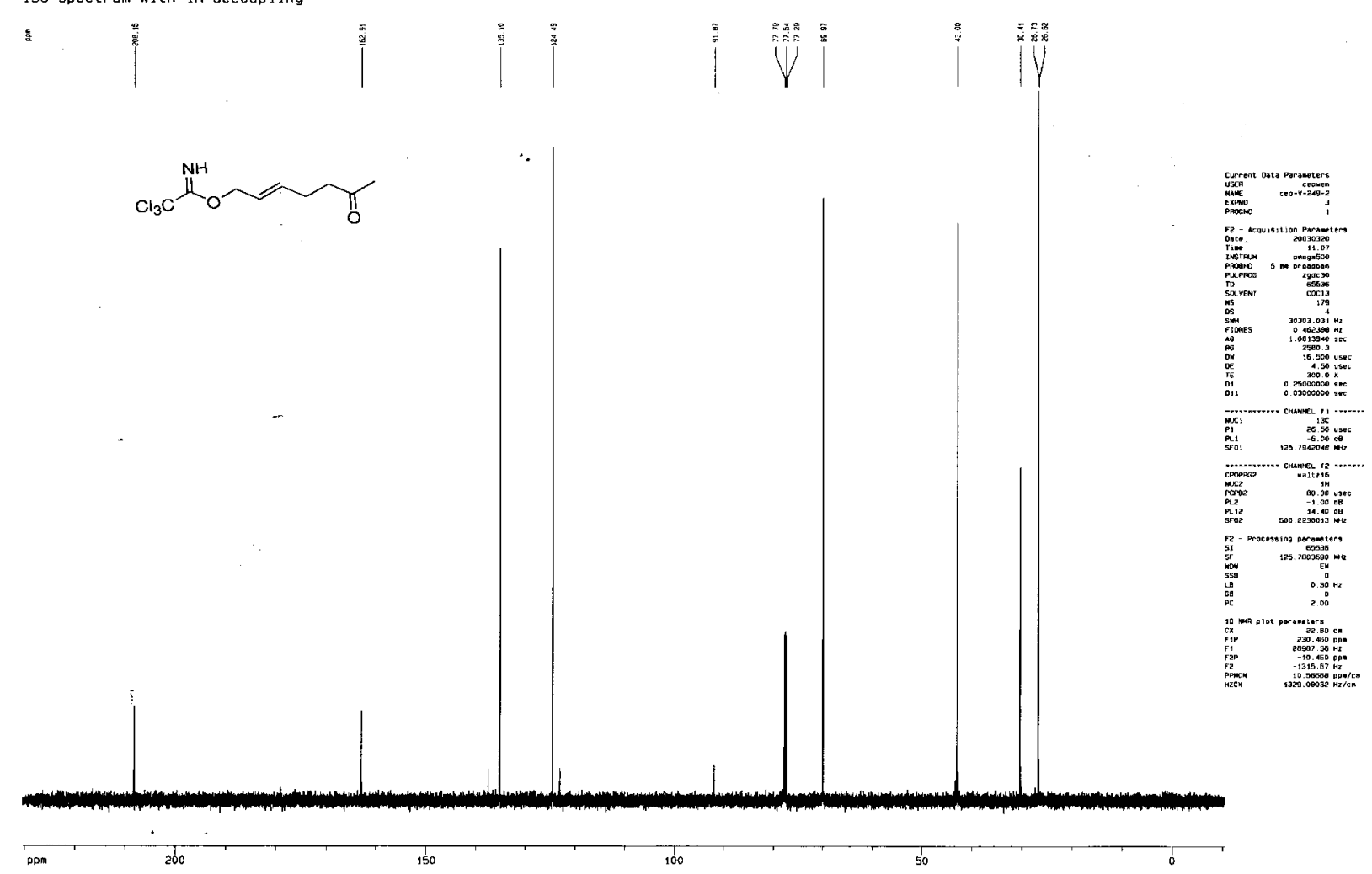


Supporting Information Anderson and Overman

1H spectrum

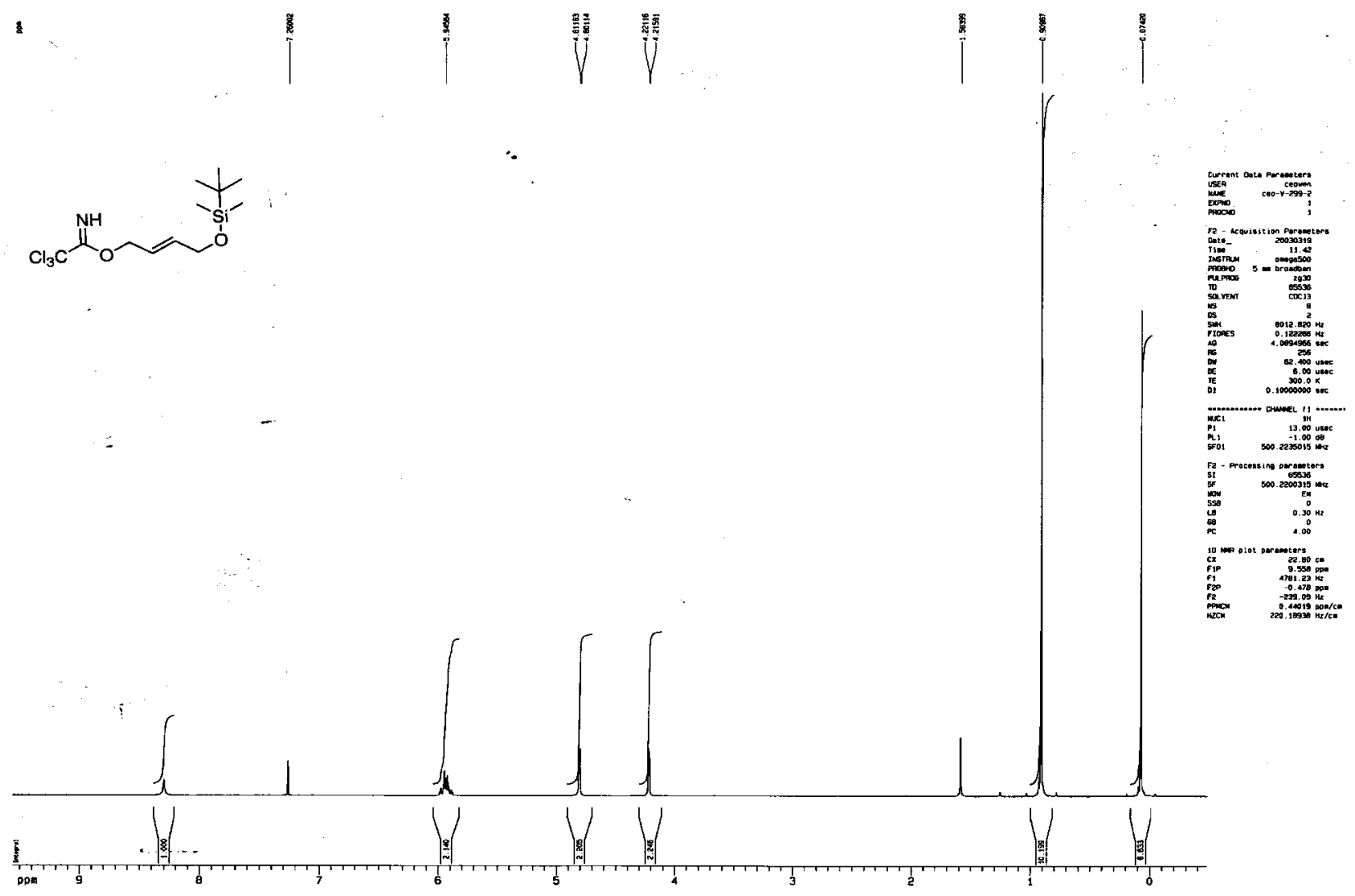

13c spectrum with iH decoupling

13c spectrum with iH decoupling
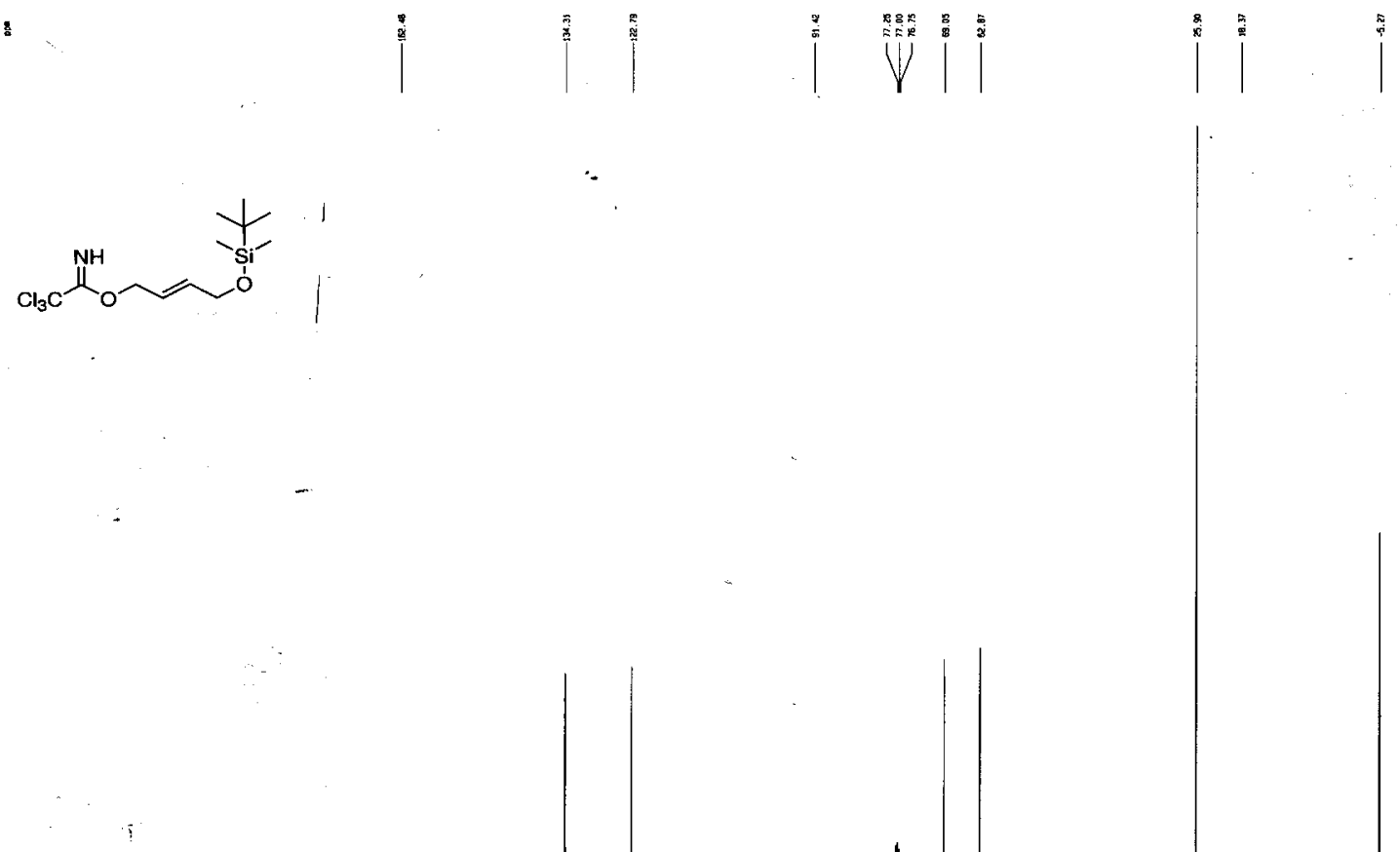
Supporting Information Anderson and Overman

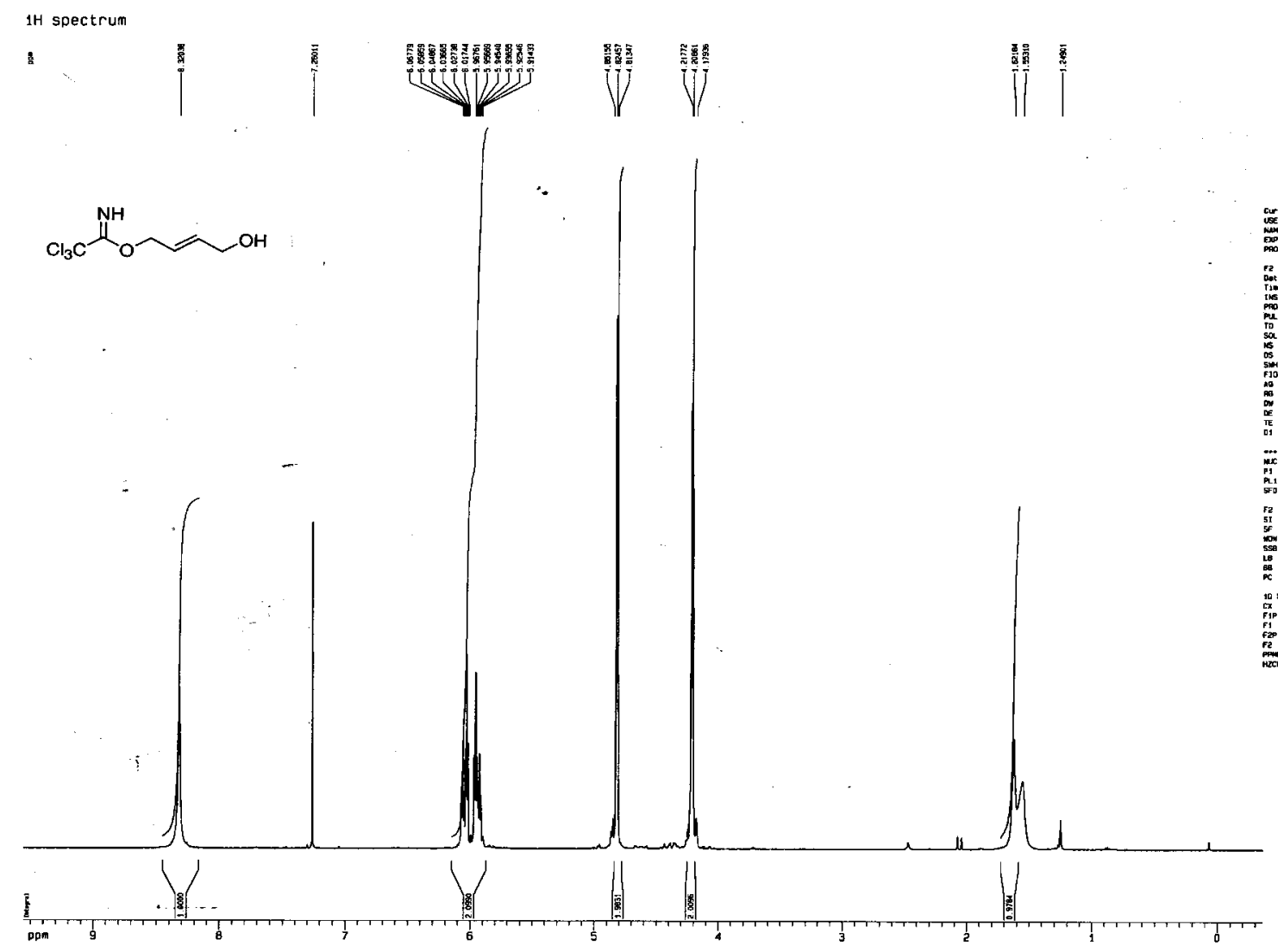

13 spectrum with $1 \mathrm{H}$ decoupling

$\Xi$



$\mathrm{Cl}_{3} \mathrm{C}^{\mathrm{NH}} \sim \mathrm{O}_{\mathrm{OH}}$

.
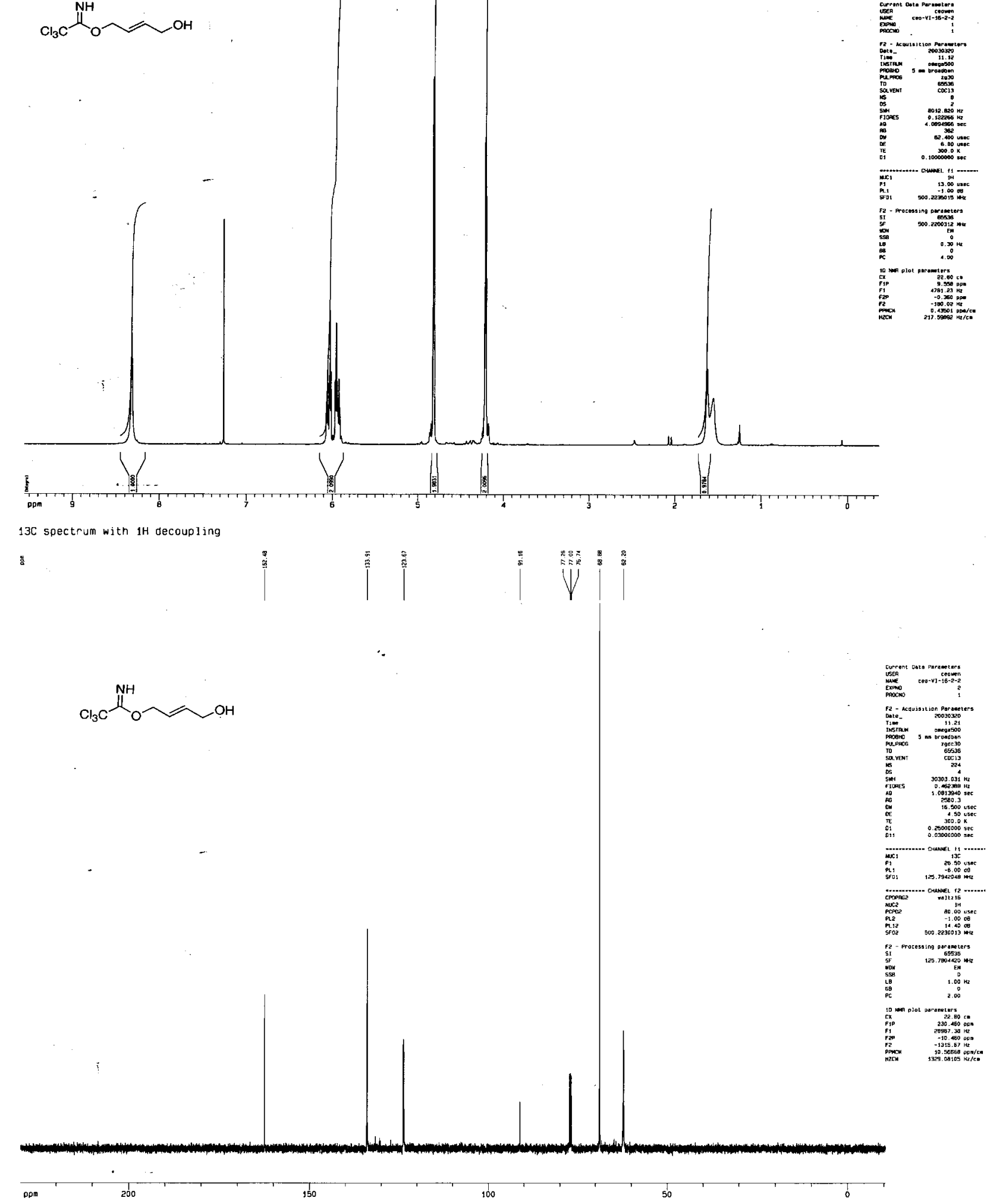
Supporting Information Anderson and Overman

$1 H$ spectrum

8



ข้อ
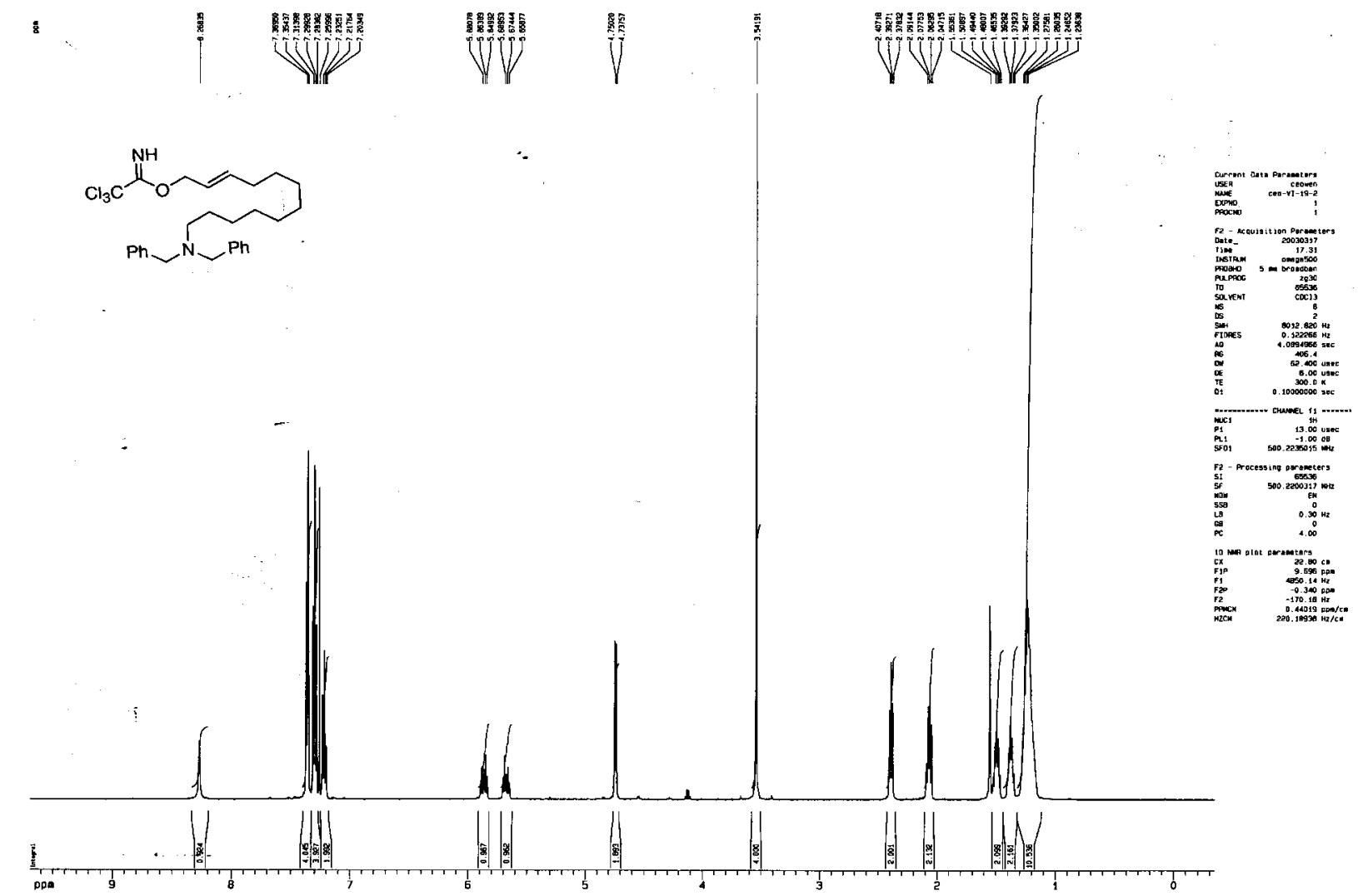

$13 \mathrm{C}$ spectrum with $1 \mathrm{H}$ decoupling

害
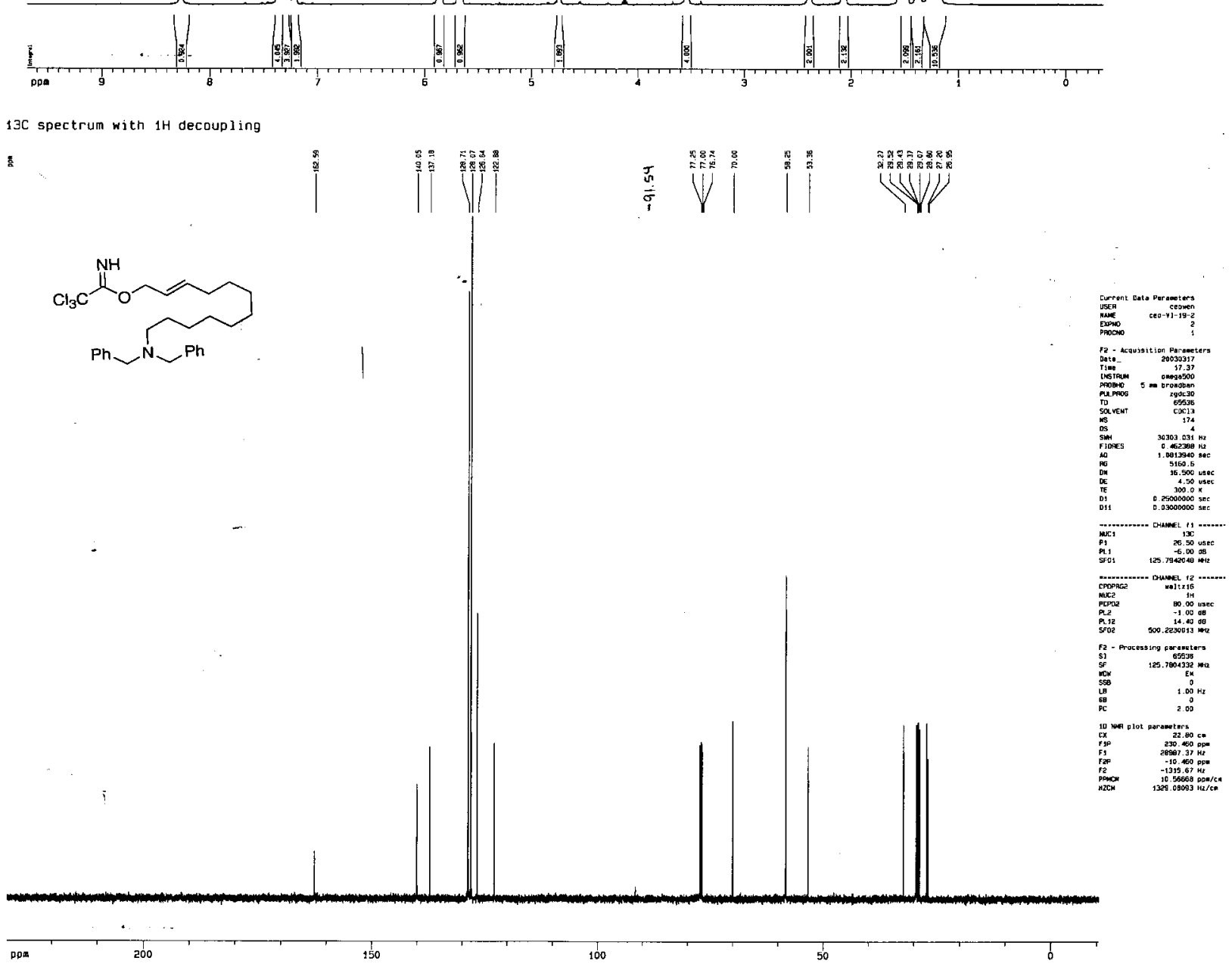
Supporting Information Anderson and Overman

$1 H$ spectrum

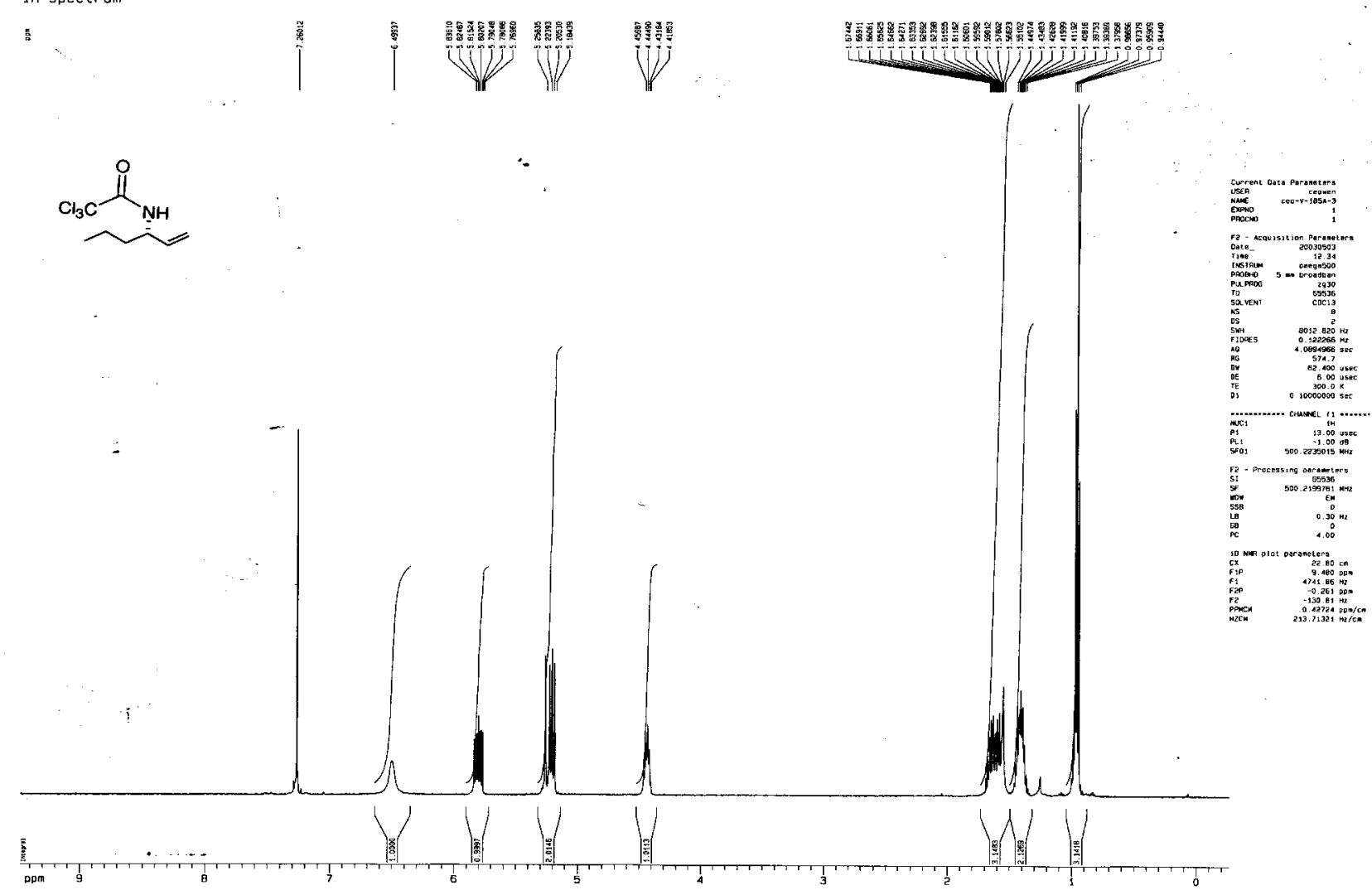

$13 \mathrm{C}$ spectrum with $1 \mathrm{H}$ decoupling

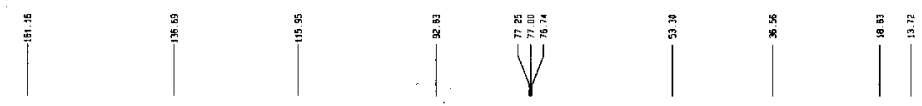

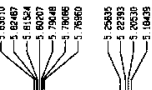

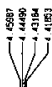

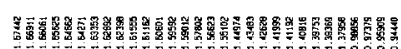

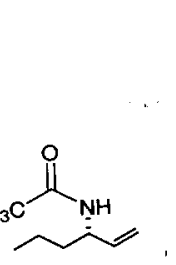


Supporting Information Anderson and Overman

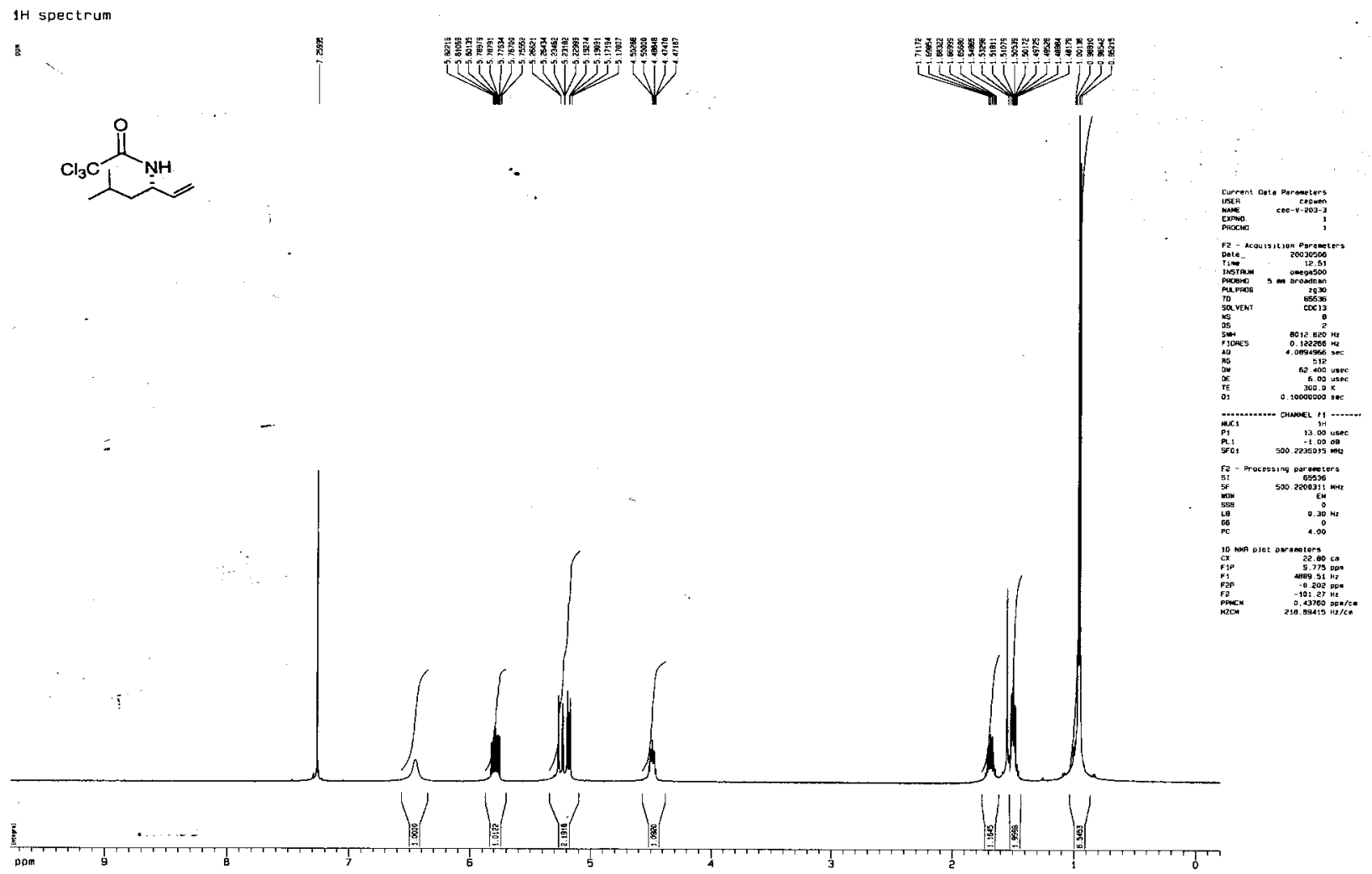

13C spectrum with $1 \mathrm{H}$ decoupling




Supporting Information Anderson and Overman
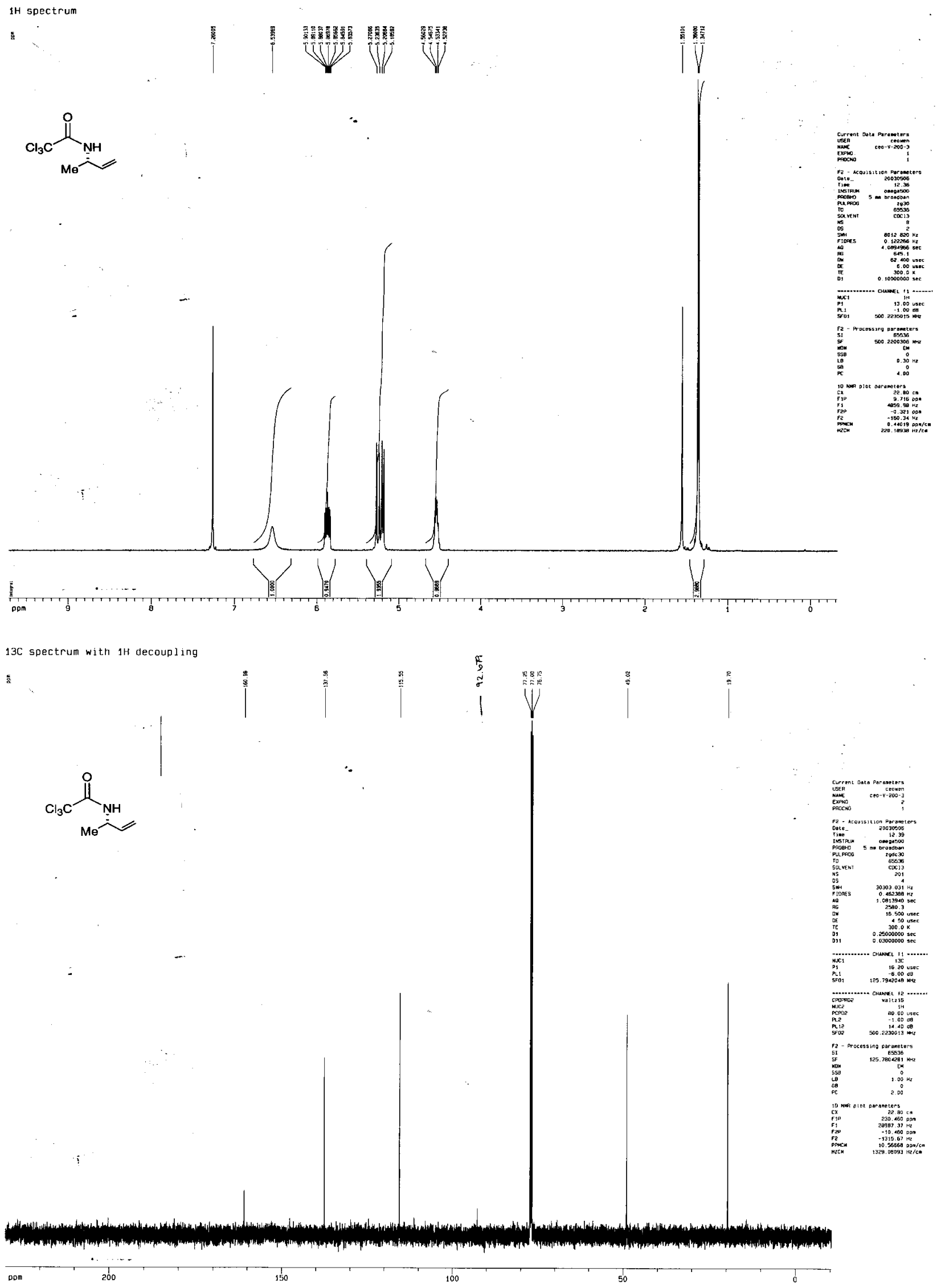
Supporting Information Anderson and Overman
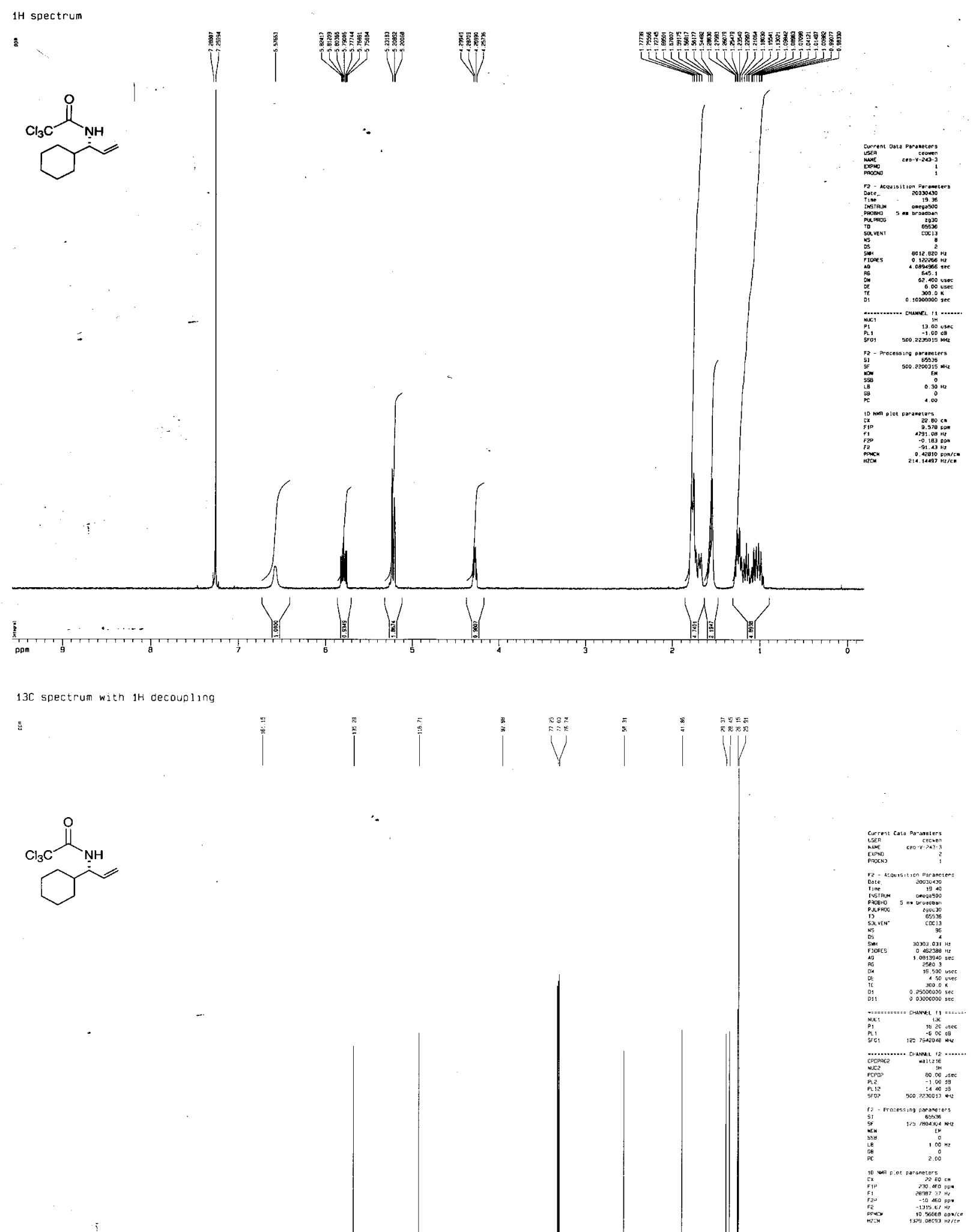
Supporting Information Anderson and Overman



13C spectrum with $1 \mathrm{H}$ decoupling

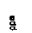
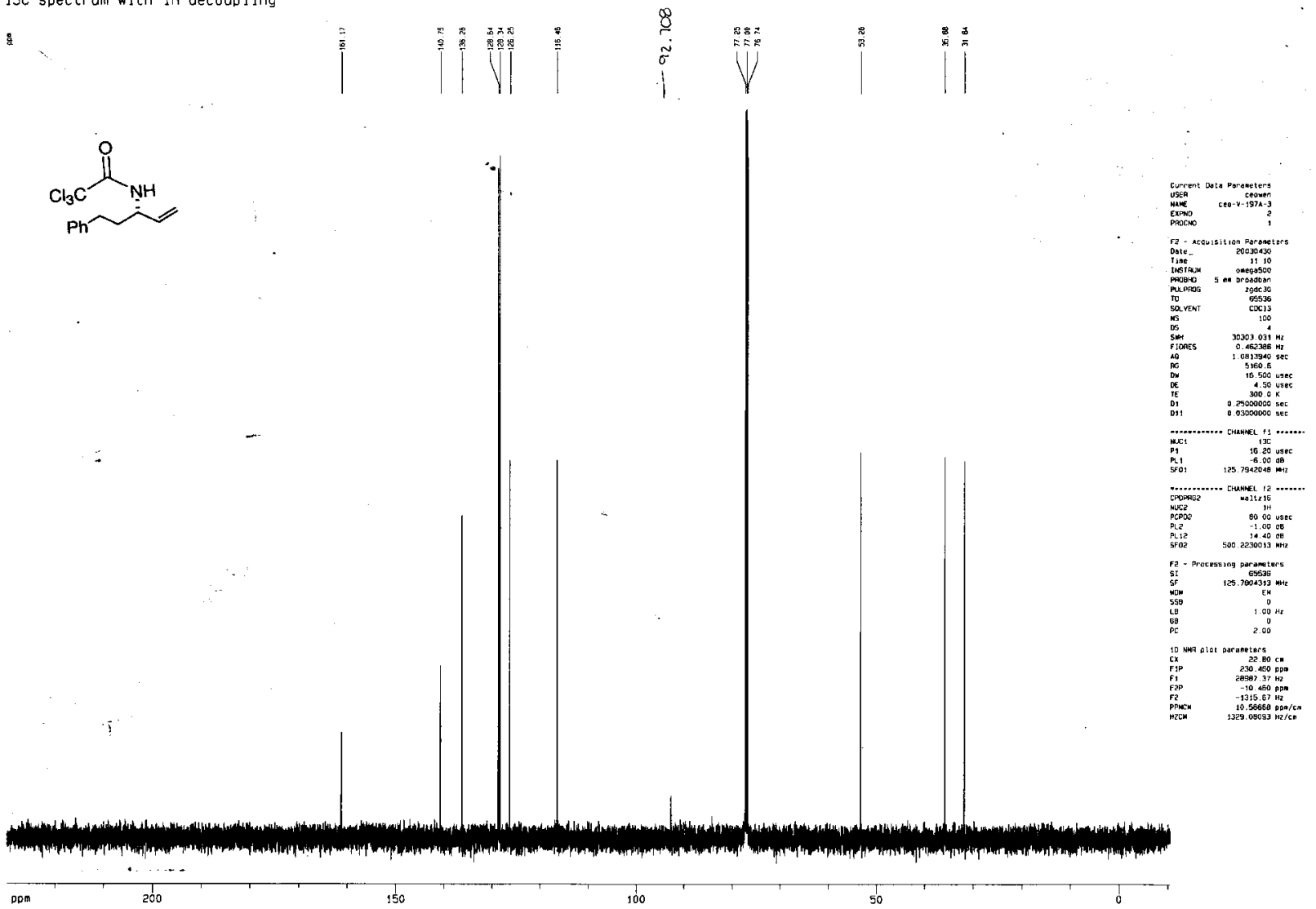
Supporting Information Anderson and Overman

$1 H$ spectrum



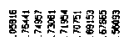




Supporting Information Anderson and Overman

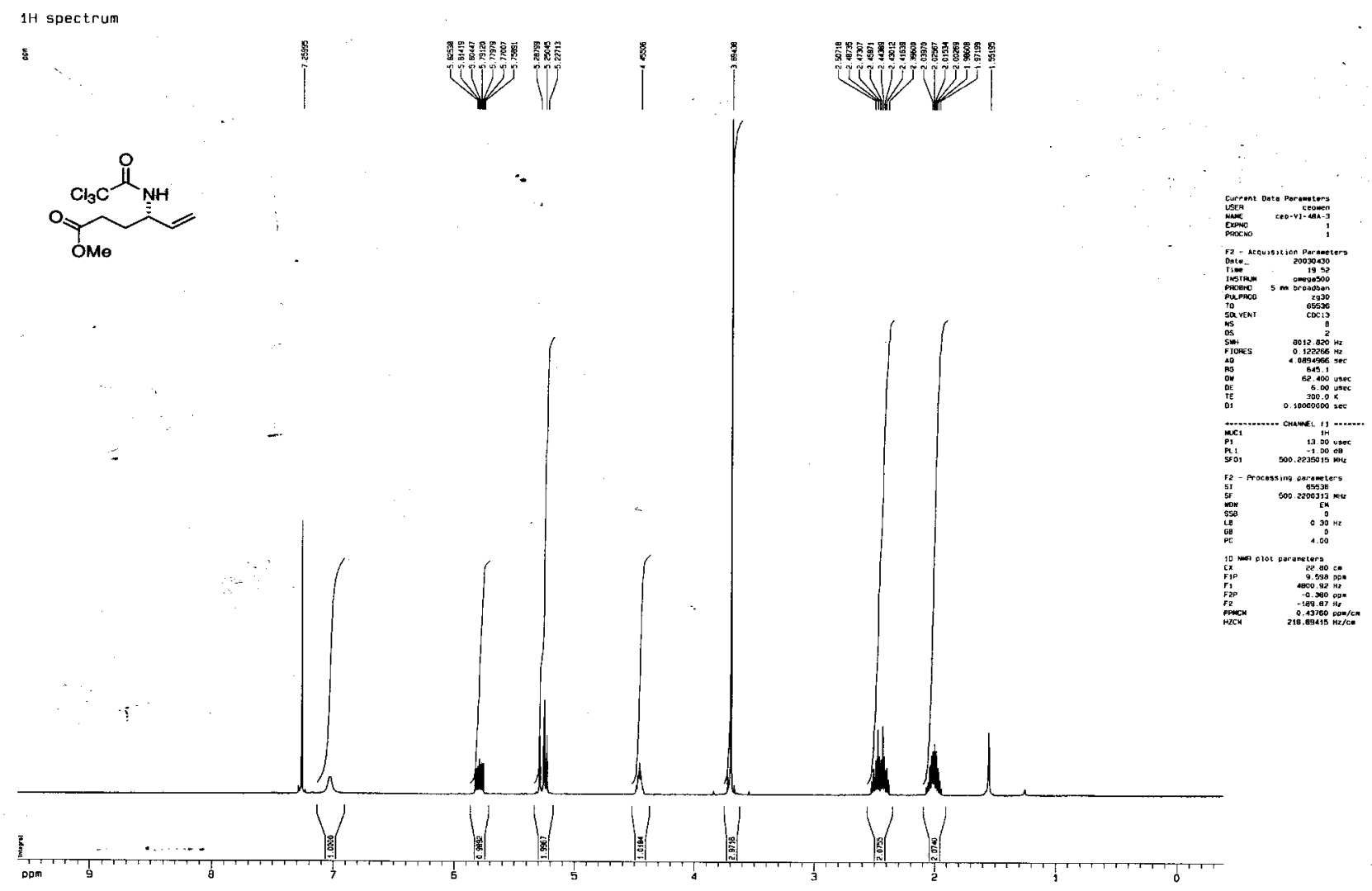

13 C spectrum with 1 H decoupling
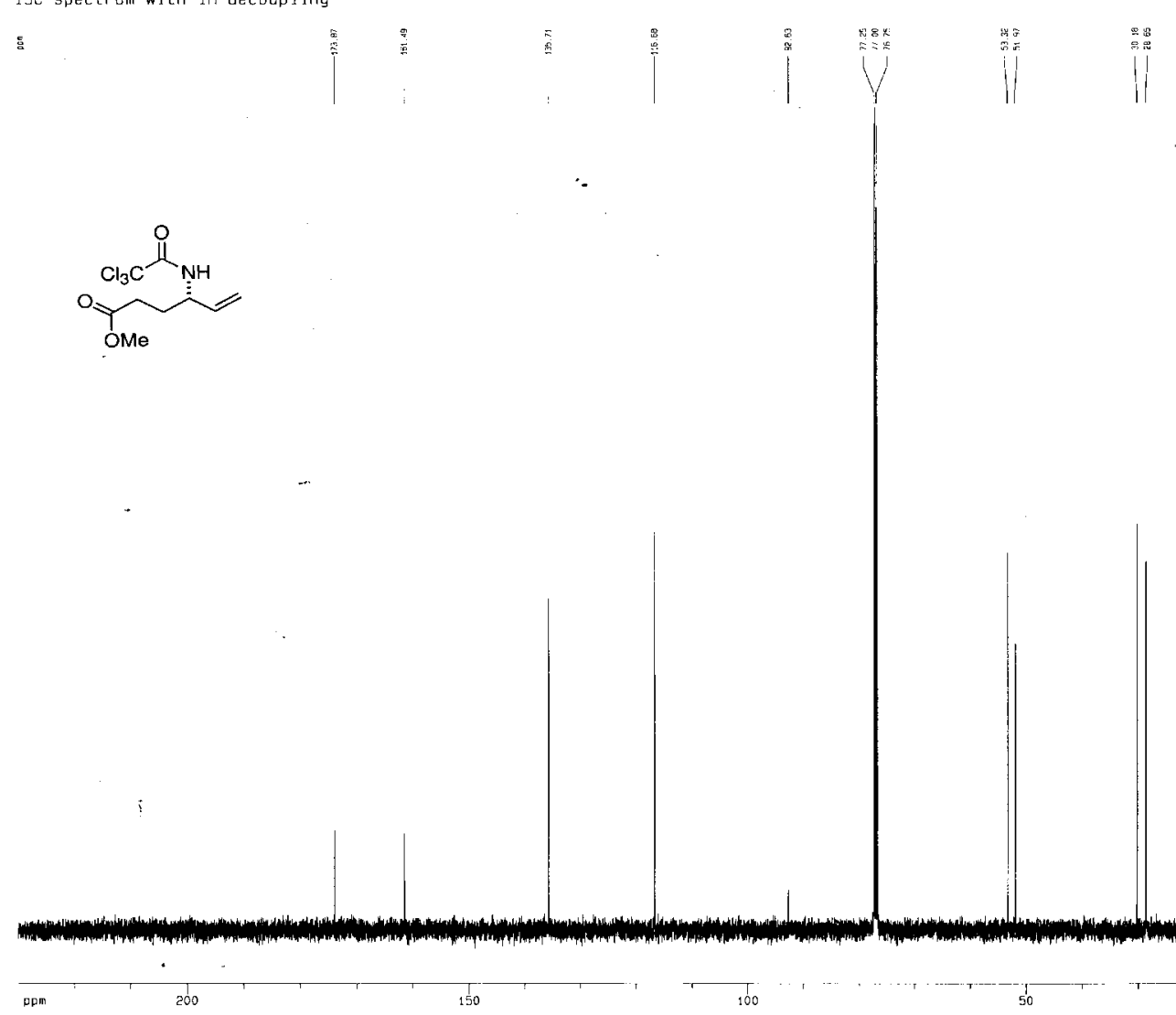

150




Supporting Information Anderson and Overman

$1 \mathrm{H}$ spectrum$$
5
$$

ह
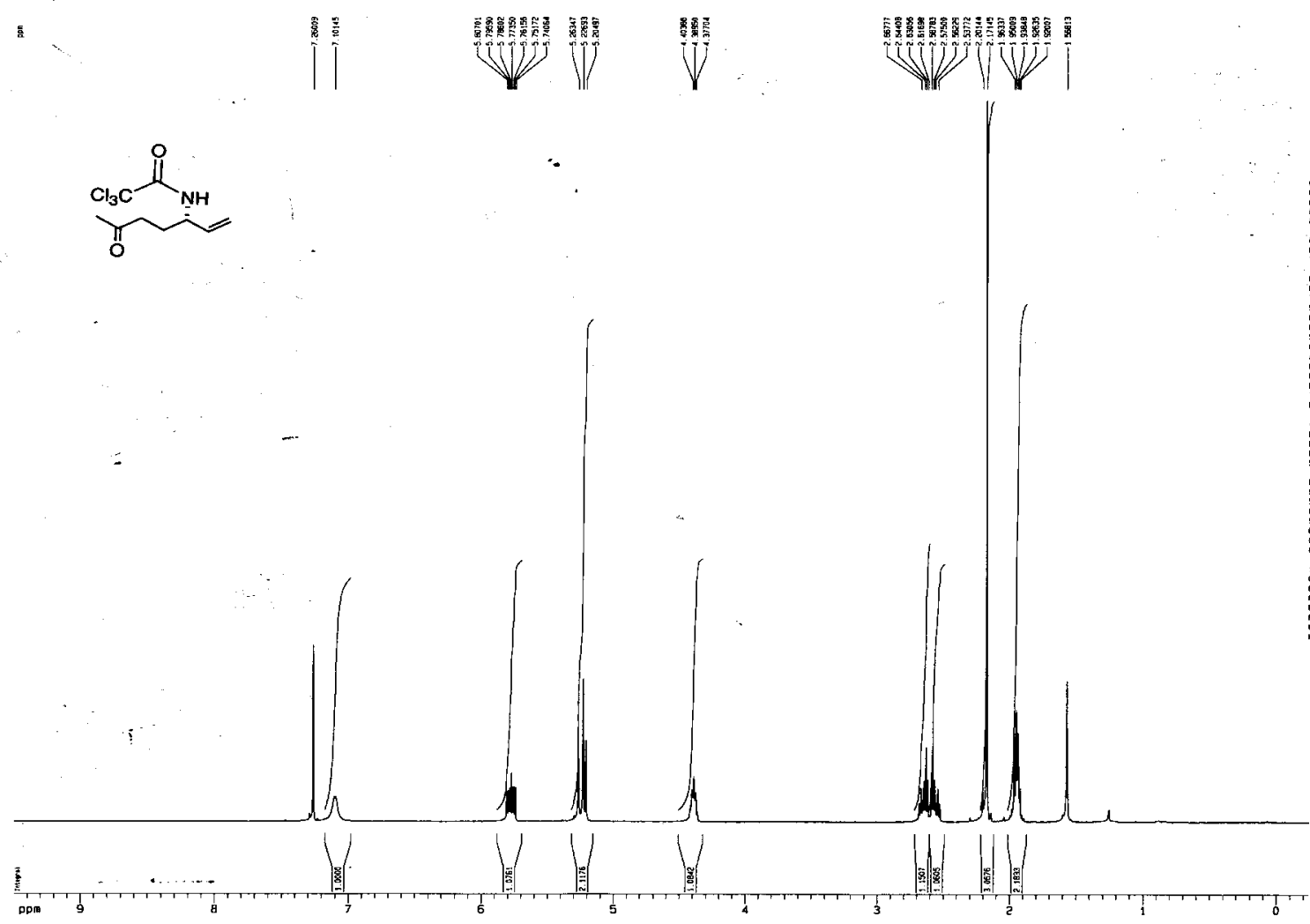

13C spectrum with $1 \mathrm{H}$ decoupling
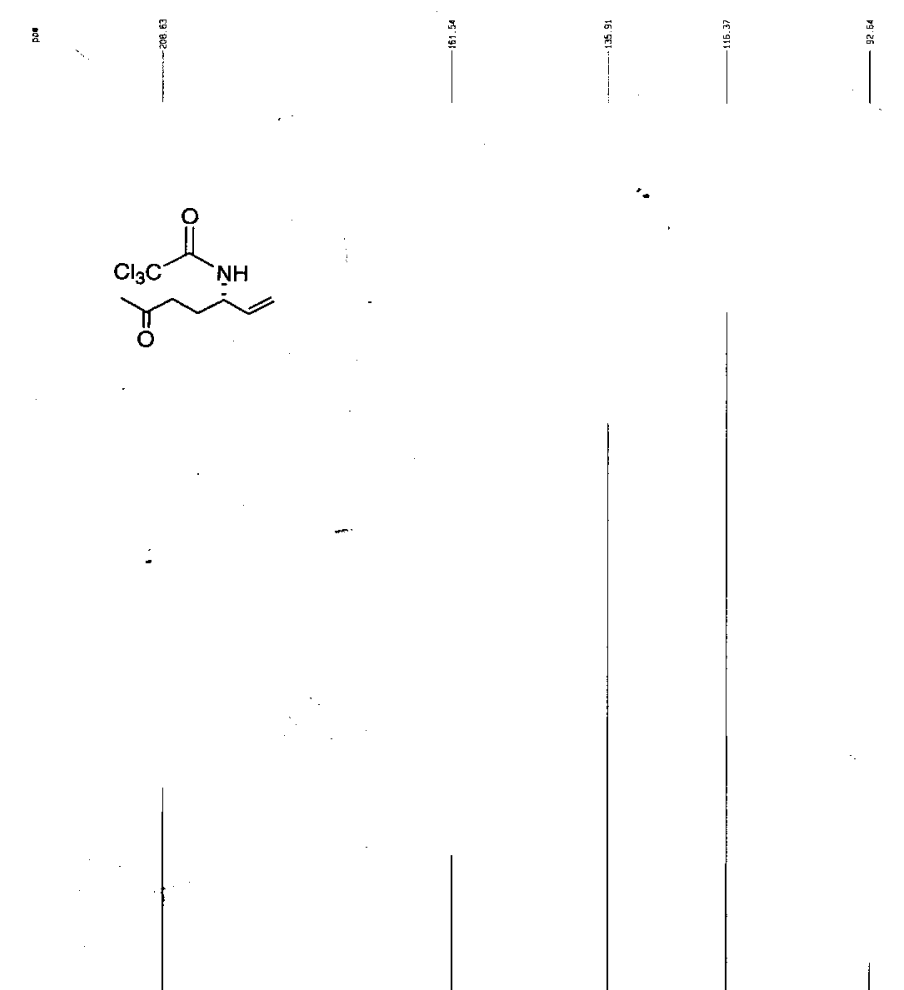

$\sqrt{1}$
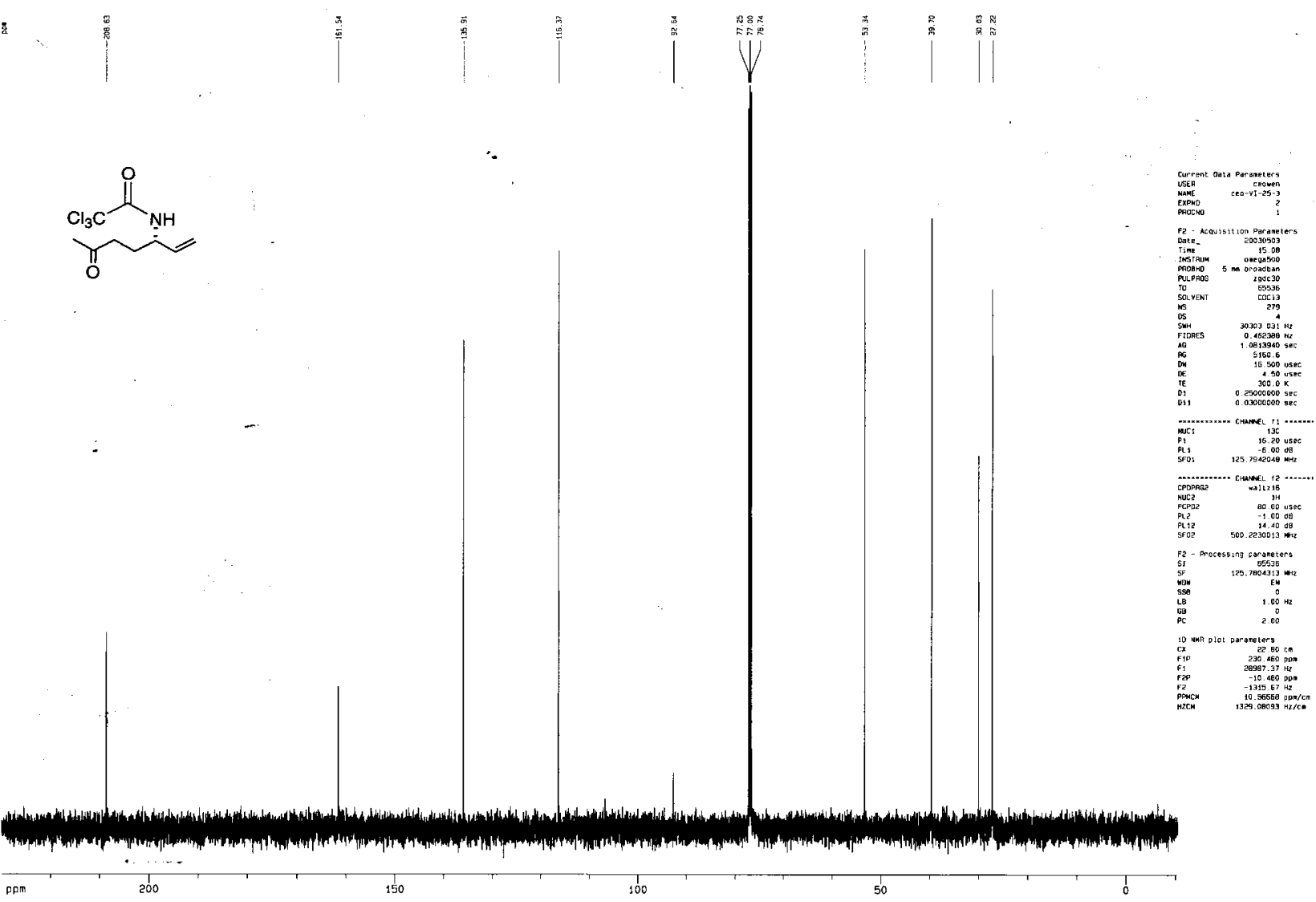
Supporting Information Anderson and Overman

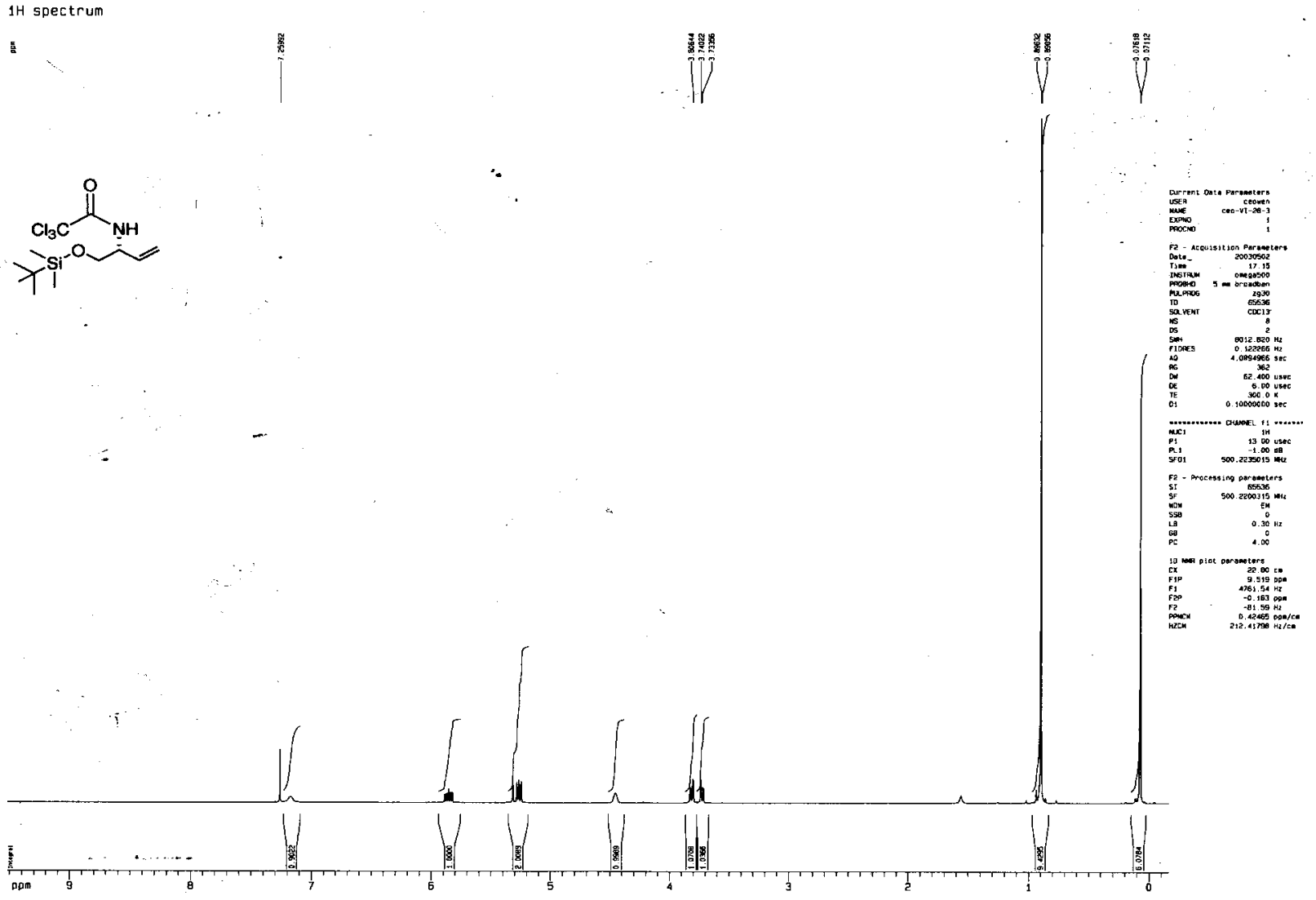

$13 \mathrm{C}$ spectrum with $1 \mathrm{H}$ decoupling

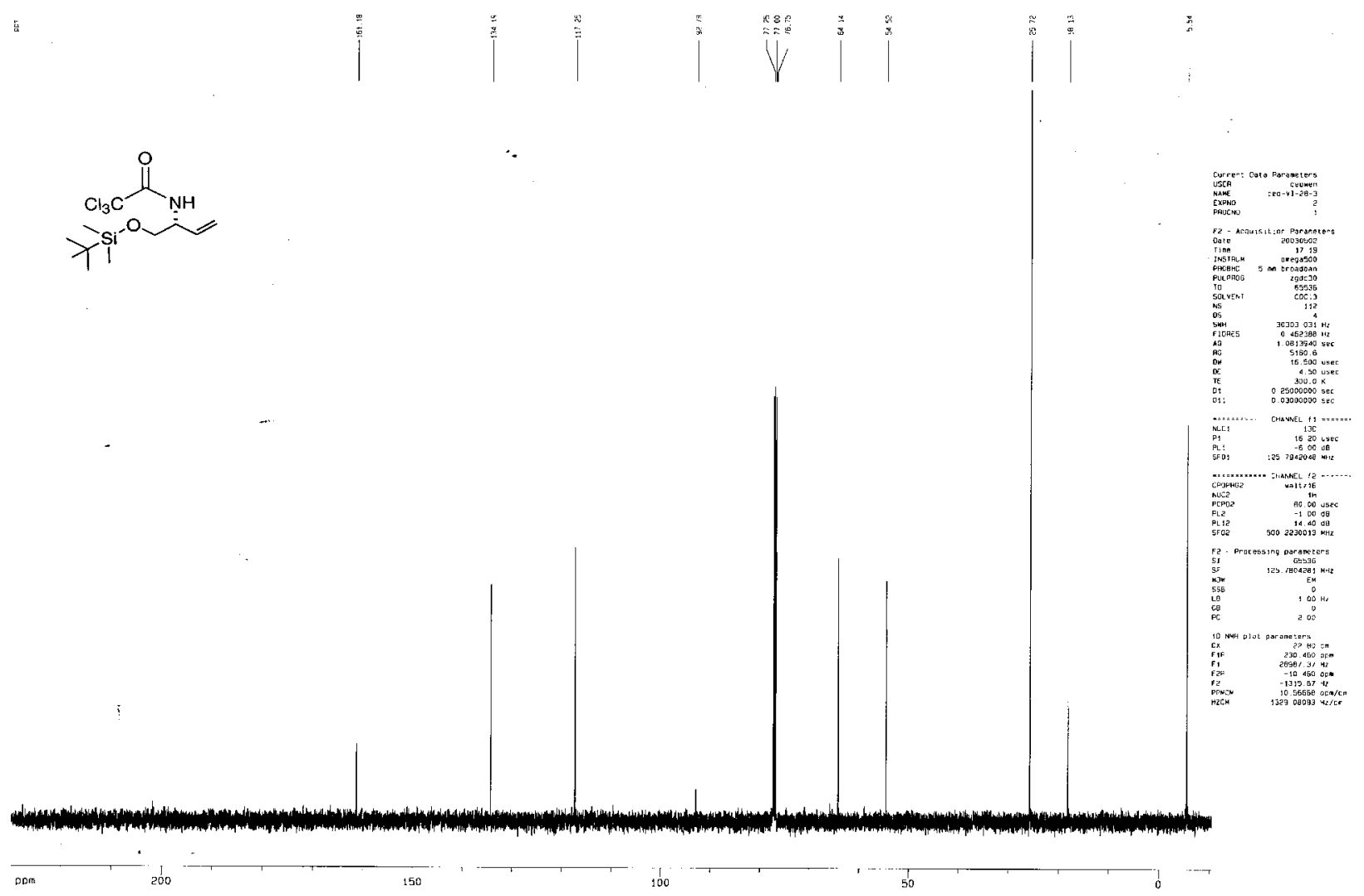


Supporting Information Anderson and Overman

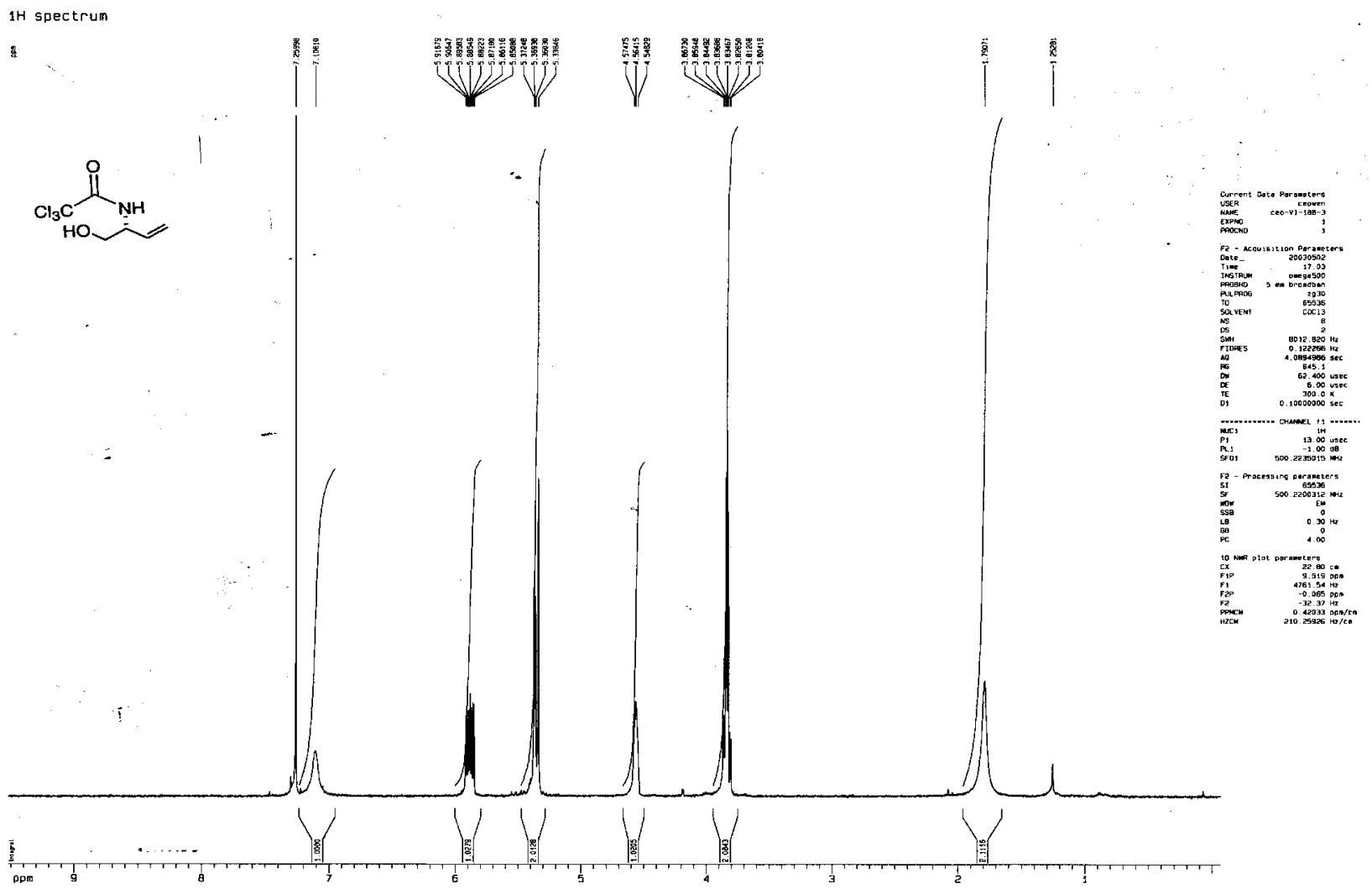

130 spectrum with $1 \mathrm{H}$ decoupling
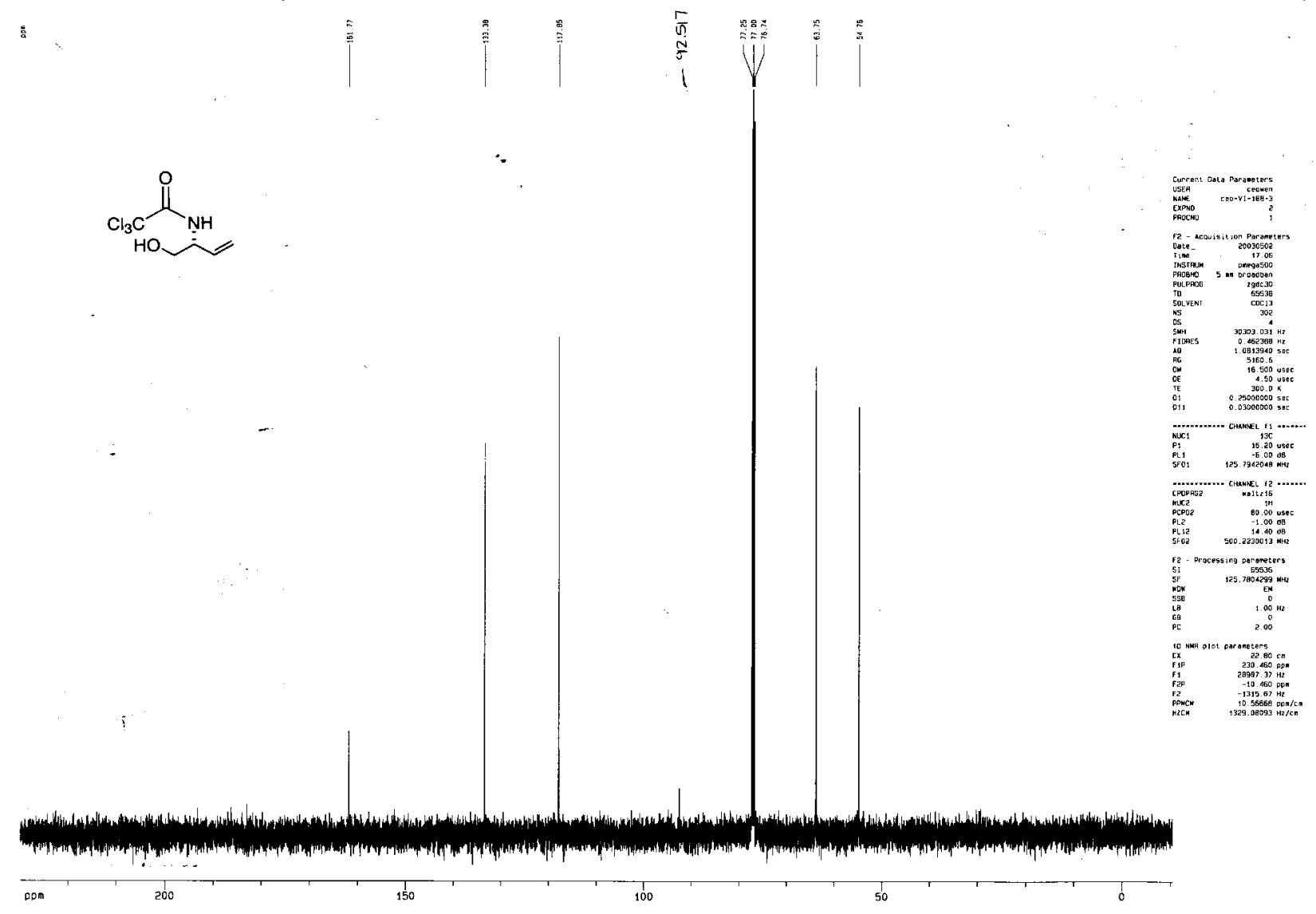
Supporting Information Anderson and Overman

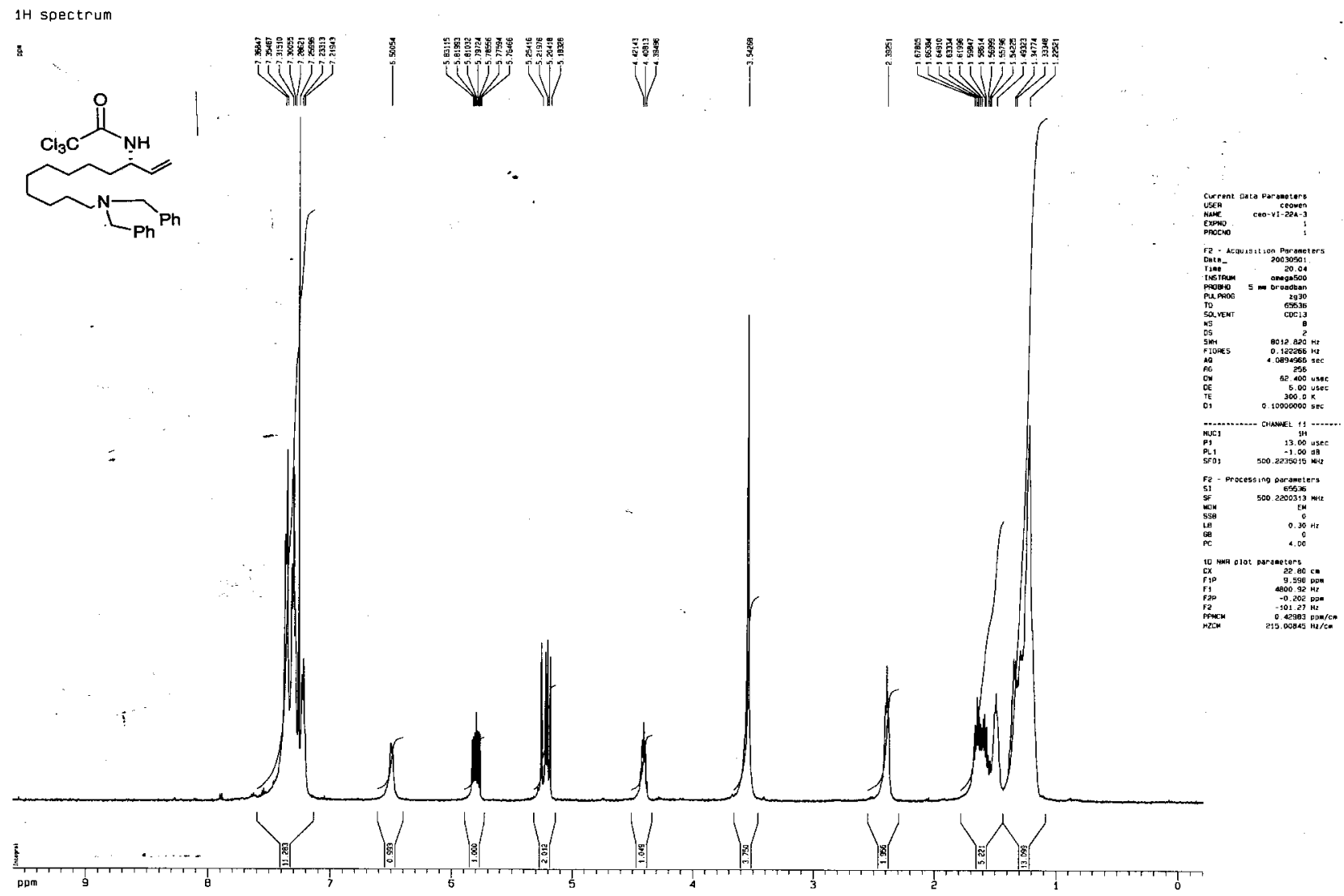

13 c spectrum with 1 decoupling
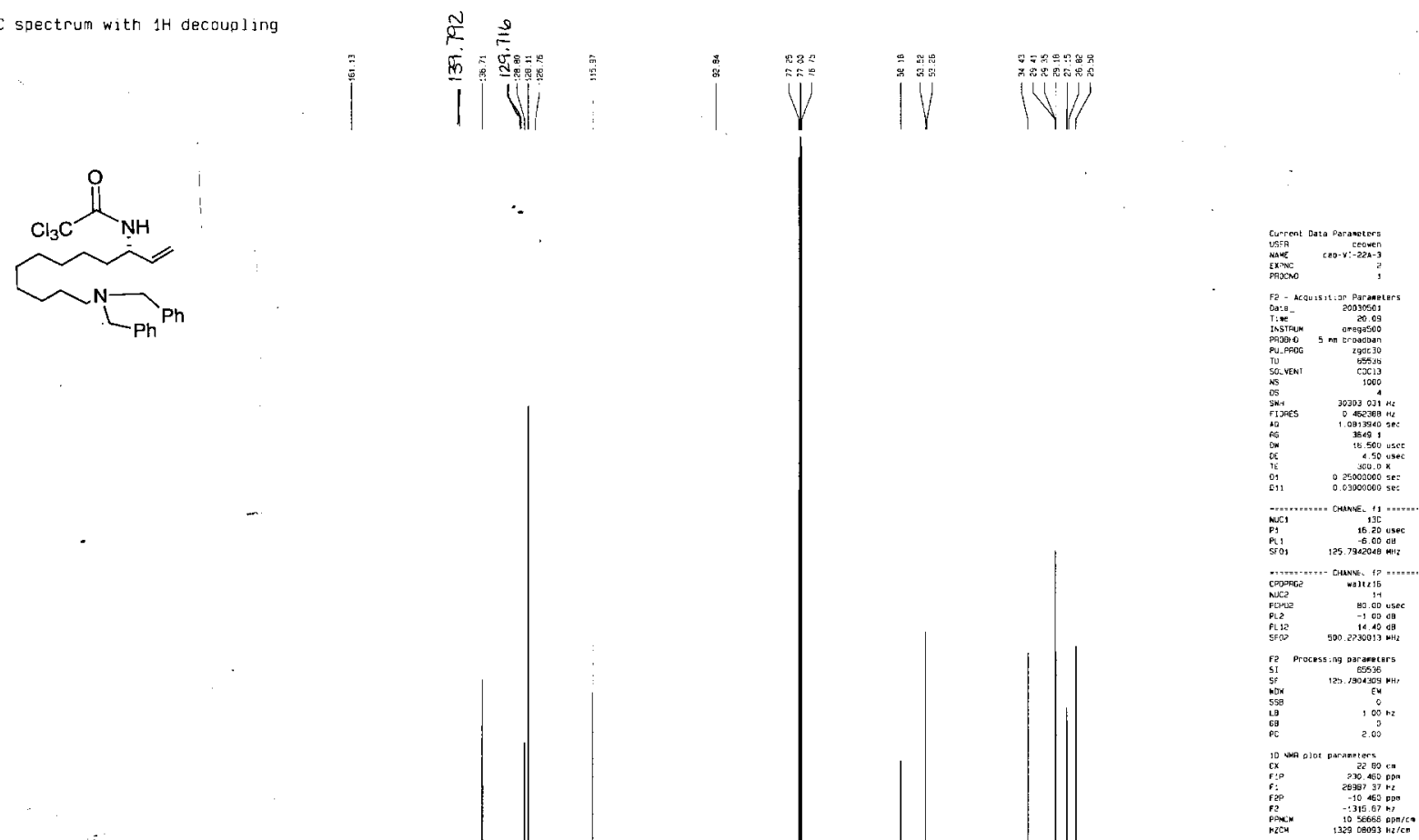
Supporting Information Anderson and Overman
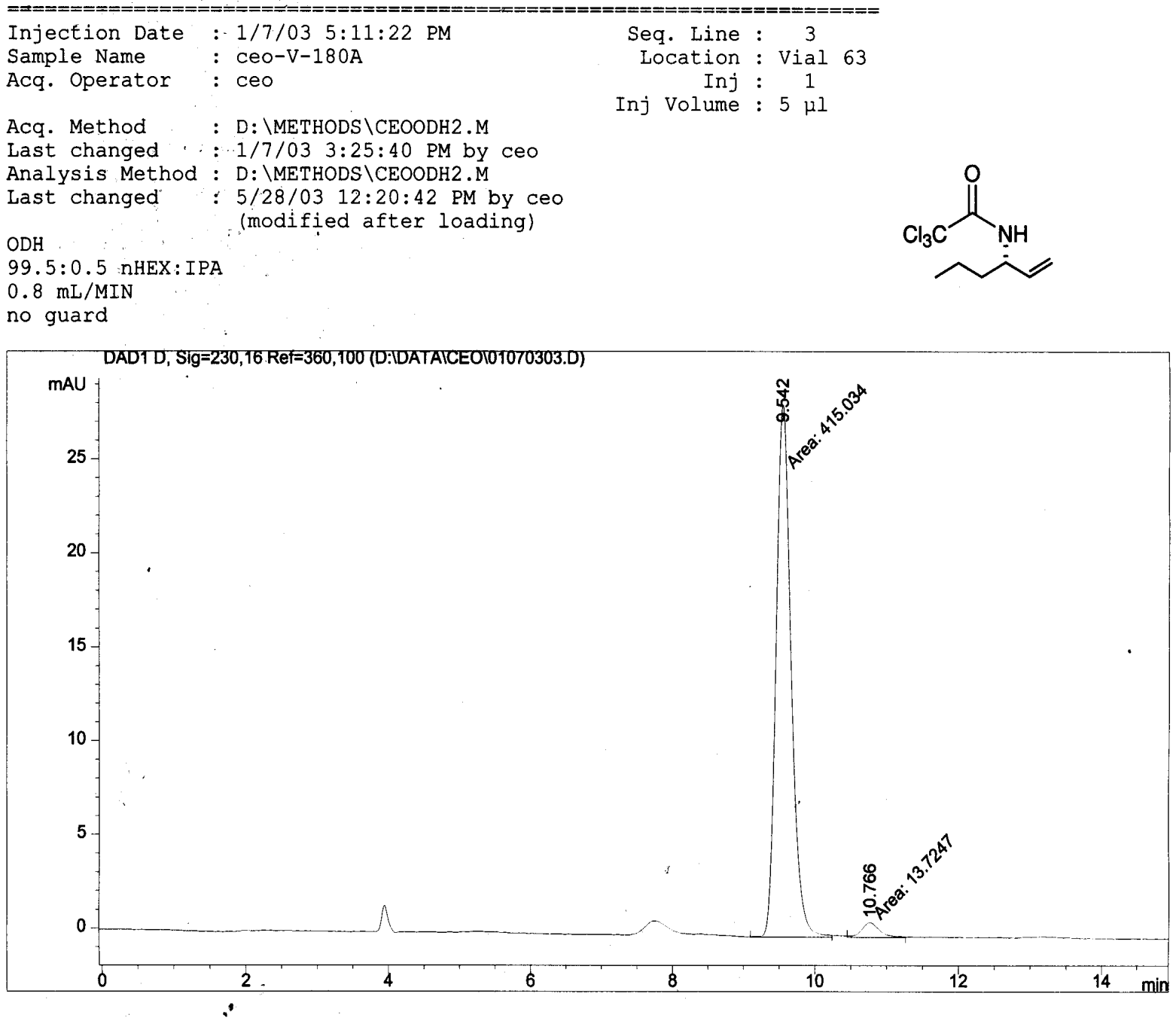

Area Percent Report

\begin{tabular}{|c|c|c|}
\hline Sorted By & : & Signal \\
\hline Multiplier & : & 1.0000 \\
\hline Dilution & : & 1.0000 \\
\hline
\end{tabular}

Use Multiplier \& Dilution Factor with ISTDs

Signal 1: DADI D, Sig=230,16 Ref $=360,100$

\begin{tabular}{|c|c|c|c|c|c|c|}
\hline $\begin{array}{c}\text { Peak } \\
\#\end{array}$ & $\begin{array}{l}\text { RetTime } \\
\text { [min]. }\end{array}$ & Type & $\begin{array}{c}\text { Width } \\
\text { [min] }\end{array}$ & $\begin{array}{c}\text { Area } \\
{\left[\mathrm{mAU}^{*} \mathrm{~s}\right]}\end{array}$ & $\begin{array}{l}\text { Height } \\
\text { [MAU] }\end{array}$ & $\begin{array}{c}\text { Area } \\
\frac{8}{\delta}\end{array}$ \\
\hline 1 &  & & & 0 & & \\
\hline $\begin{array}{l}1 \\
2\end{array}$ & $\begin{array}{r}9.542 \\
10.766\end{array}$ & $\begin{array}{l}\text { MM } \\
\text { MM }\end{array}$ & $\begin{array}{l}0.2436 \\
0.2919\end{array}$ & $\begin{array}{r}415.03391 \\
13.72468\end{array}$ & $\begin{array}{r}28.40023 \\
7.83625 e-1\end{array}$ & $\begin{array}{r}96.7990 \\
3.2010\end{array}$ \\
\hline
\end{tabular}

Results obtained with enhanced integrator! 
Supporting Information Anderson and Overman
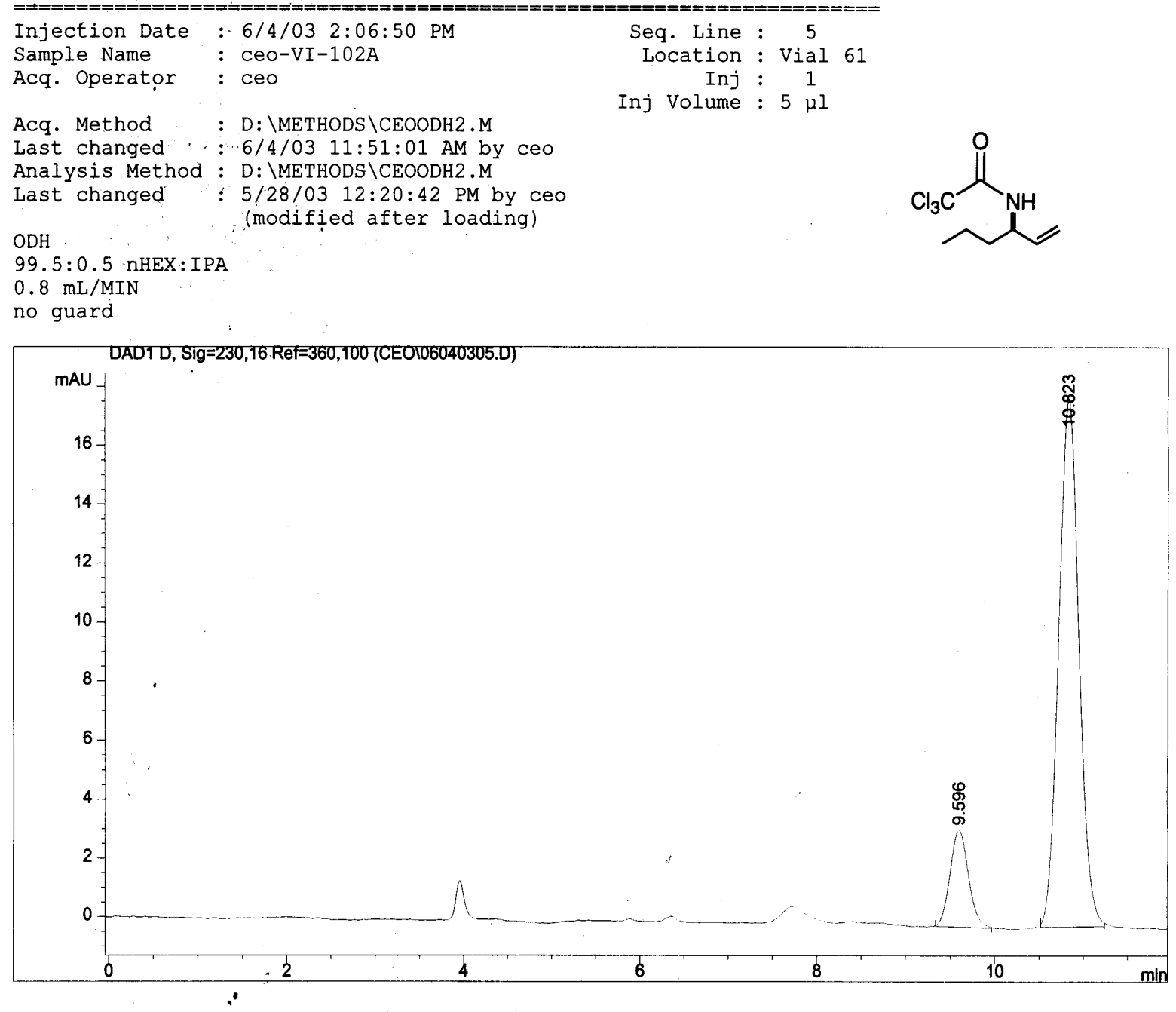

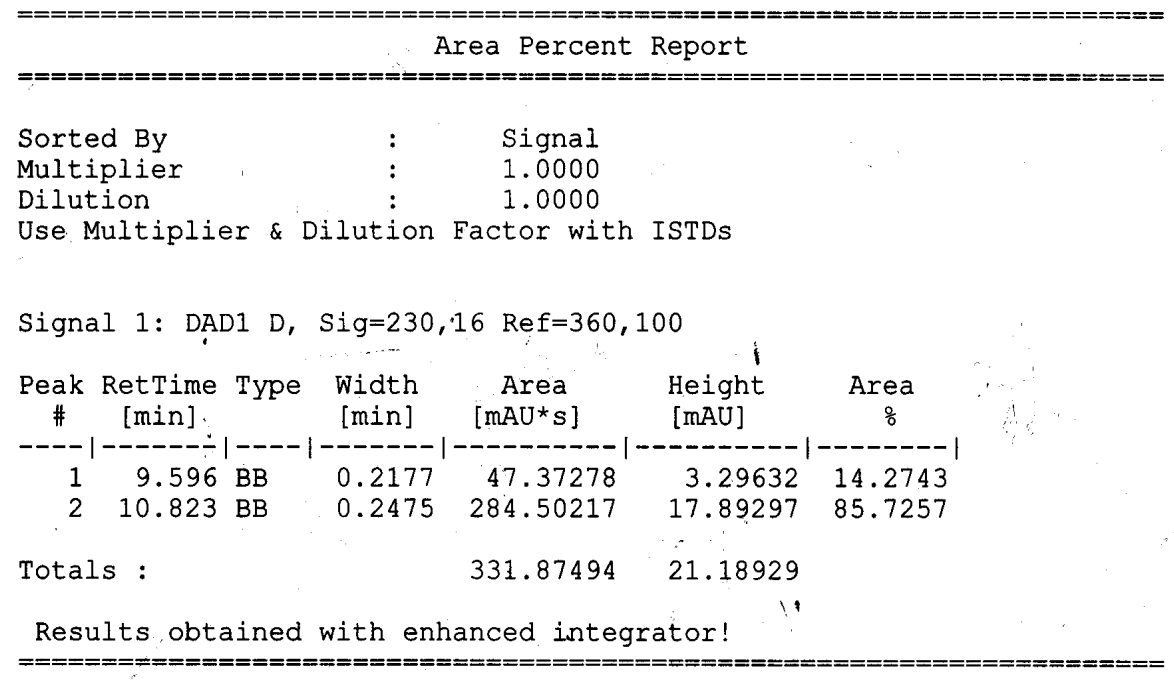

$\star \star \star$ End of Report $\star \star \star$ 
Supporting Information Anderson and Overman

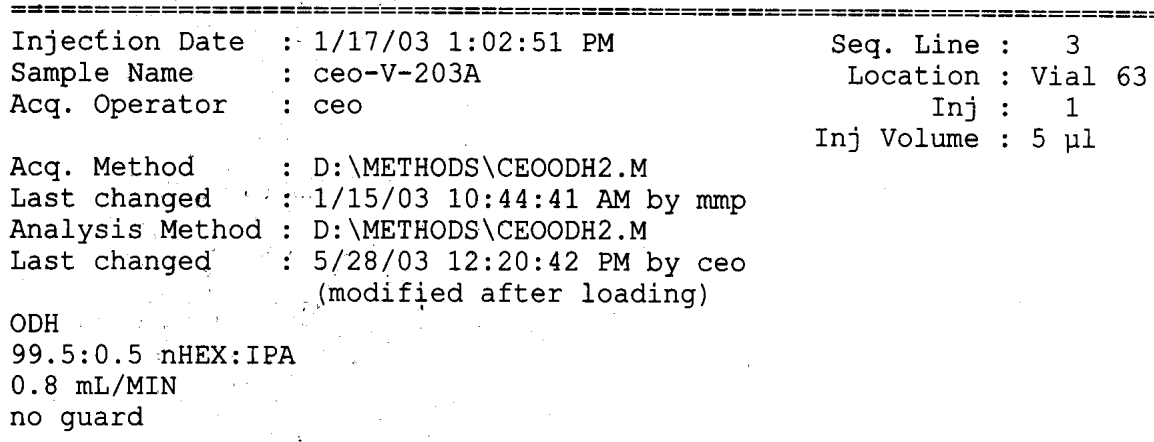

Seq. Line : 3

Location : Vial 63

Inj : 1

Inj Volume : $5 \mu \mathrm{l}$

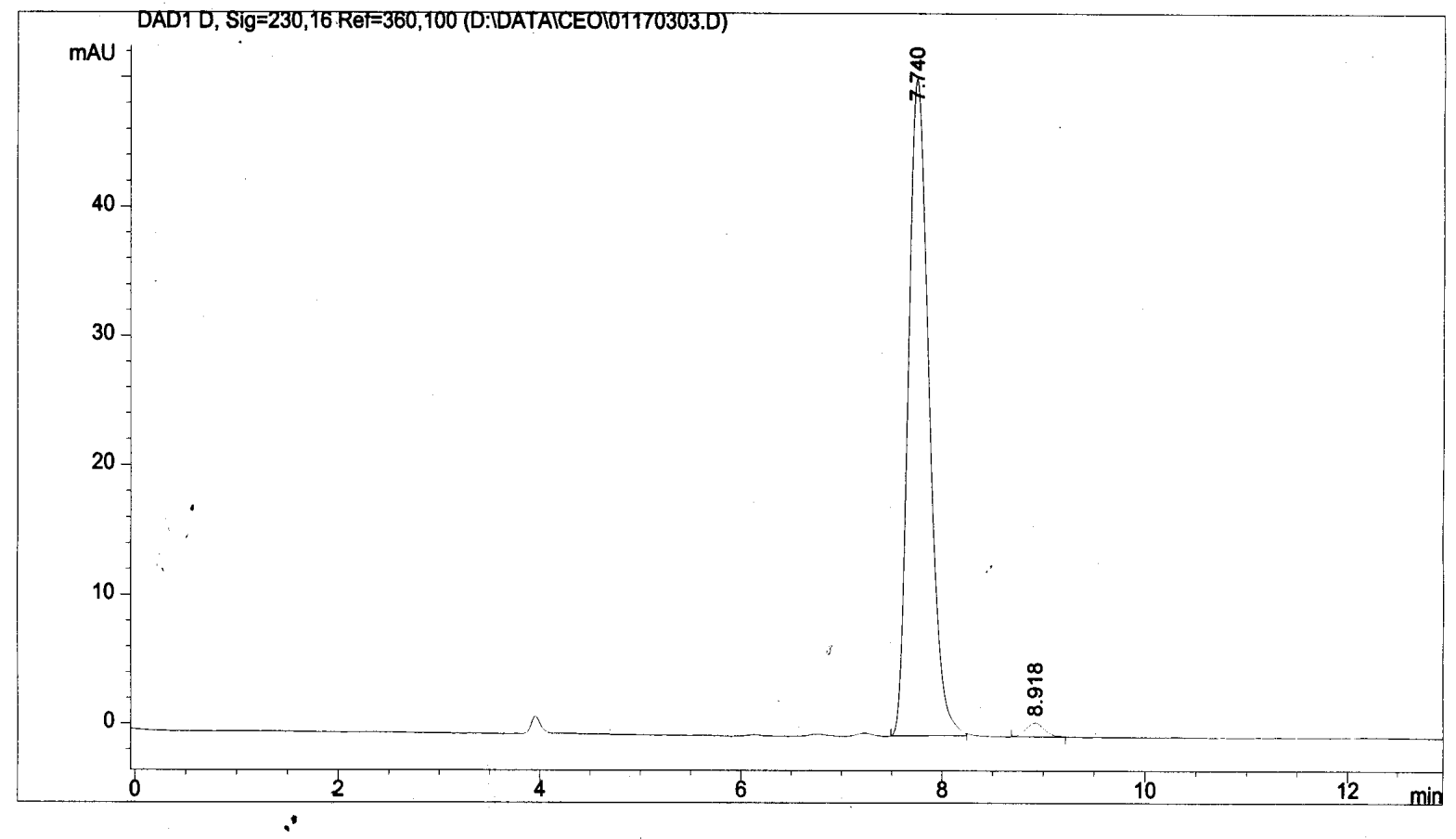

Area Percent Report




Supporting Information Anderson and Overman

Data File C: \HPCHEM $\backslash 1 \backslash D A T A \backslash C E O \backslash 06040303 . D$



99.5:0.5 nHEX:IPA

$0.8 \mathrm{~mL} / \mathrm{MIN}$

no guard

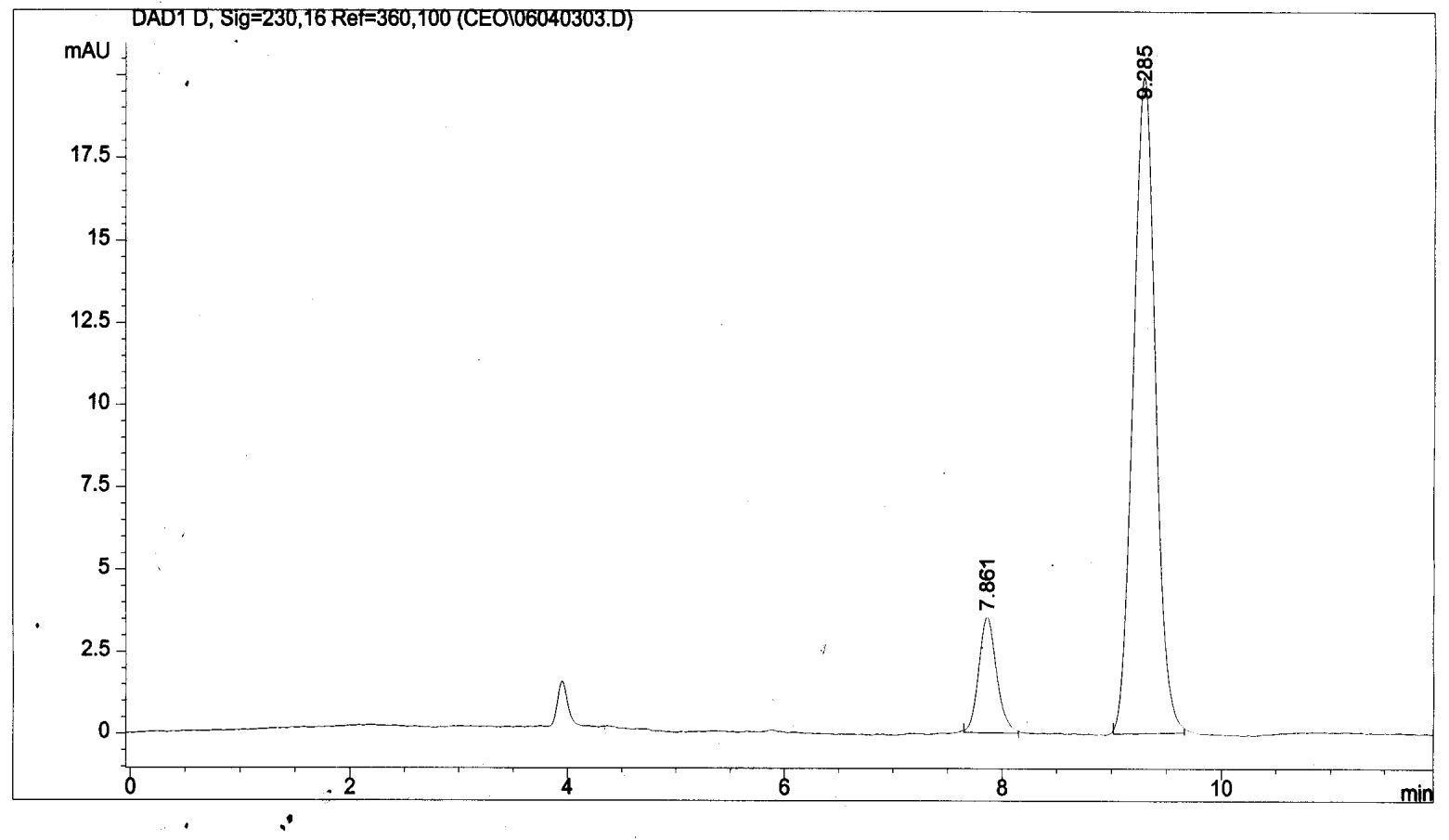

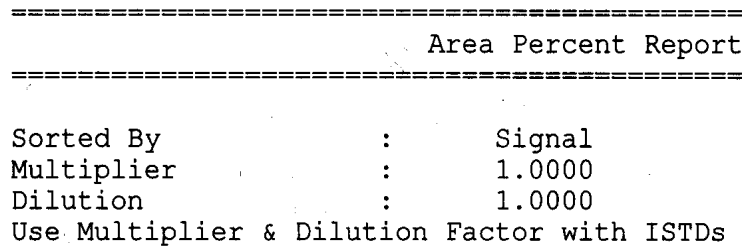

Signal 1: DAD1 D, Sig=230, 16 Ref $=360,100$

\begin{tabular}{|c|c|c|c|c|c|c|}
\hline $\begin{array}{c}\text { Peak } \\
\#\end{array}$ & $\begin{array}{l}\text { RetTime } \\
\text { [min]. }\end{array}$ & Type & $\begin{array}{l}\text { Width } \\
\text { [min] }\end{array}$ & $\begin{array}{c}\text { Area } \\
{\left[\mathrm{mAU}{ }^{*} \mathrm{~s}\right]}\end{array}$ & $\begin{array}{l}\text { Height } \\
\text { [MAU] }\end{array}$ & $\begin{array}{c}\text { Area } \\
\frac{\circ}{0}\end{array}$ \\
\hline 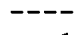 & -- & . & . & -1 & 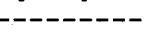 & --- \\
\hline $\begin{array}{r}.1 \\
2\end{array}$ & $\begin{array}{l}7.861 \\
9.285\end{array}$ & $\begin{array}{l}\mathrm{BB} \\
\mathrm{BB}\end{array}$ & $\begin{array}{l}0.1753 \\
0.2188\end{array}$ & $\begin{array}{r}40.20476 \\
281.61255\end{array}$ & $\begin{array}{r}3.51793 \\
19.94164\end{array}$ & $\begin{array}{l}12.4930 \\
87.5070\end{array}$ \\
\hline
\end{tabular}

Totals : $\quad 321.81731 \quad 23.45957$

Results obtained with enhanced integrator! 
Supporting Information Anderson and Overman
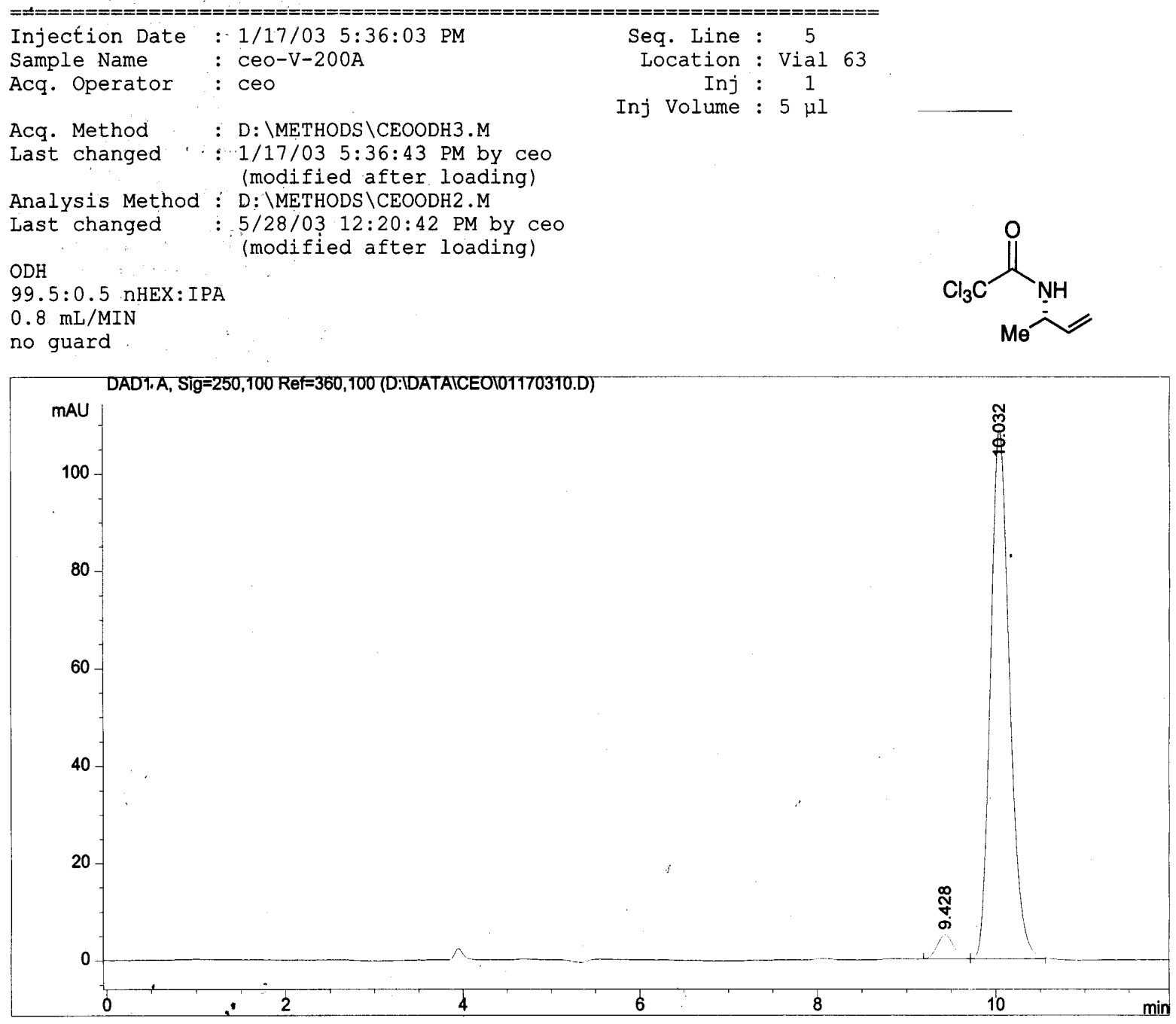

Area Percent Report

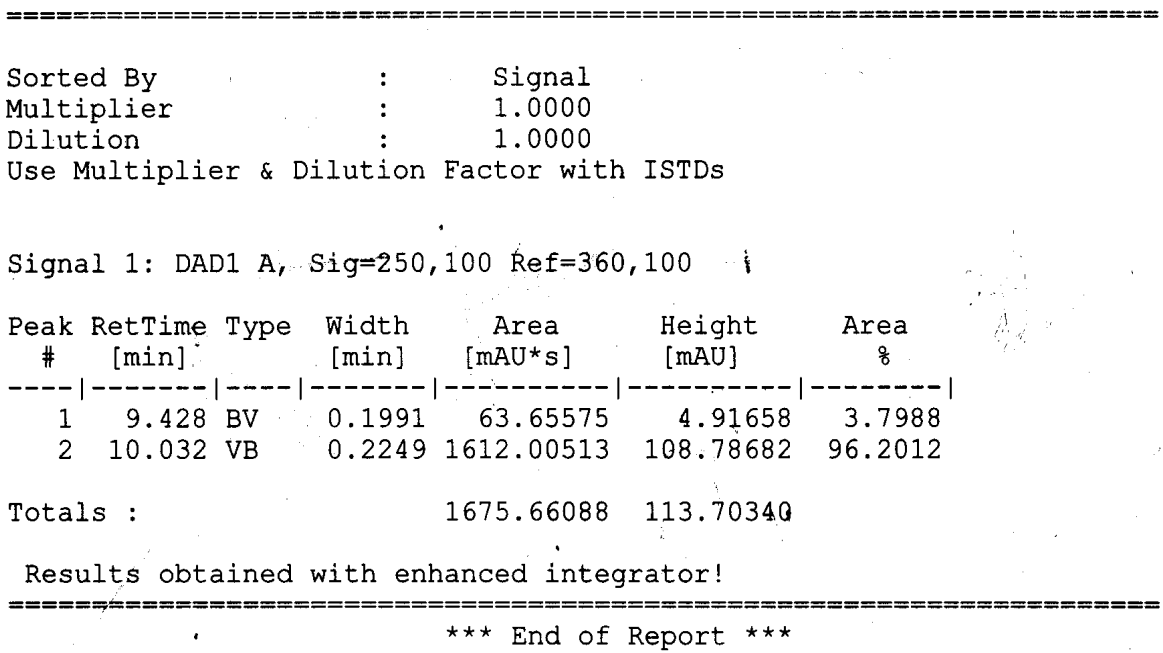


Supporting Information Anderson and Overman

Data File D: \DATA \CEO $\backslash 02150302 . D$

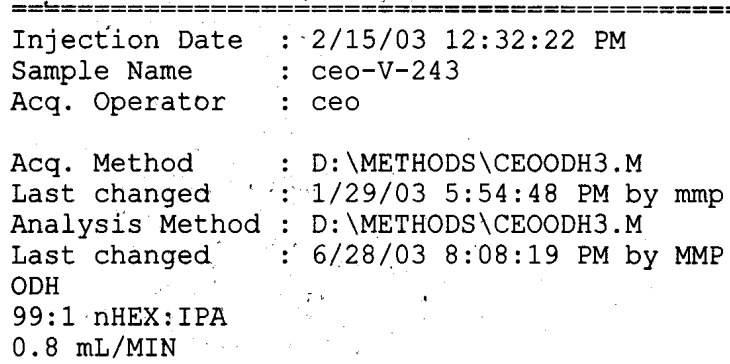

$0.8 \mathrm{~mL} / \mathrm{MIN}$

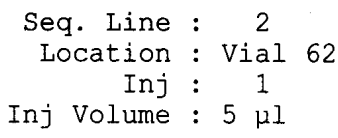

Inj Volume : $5 \mu \mathrm{l}$
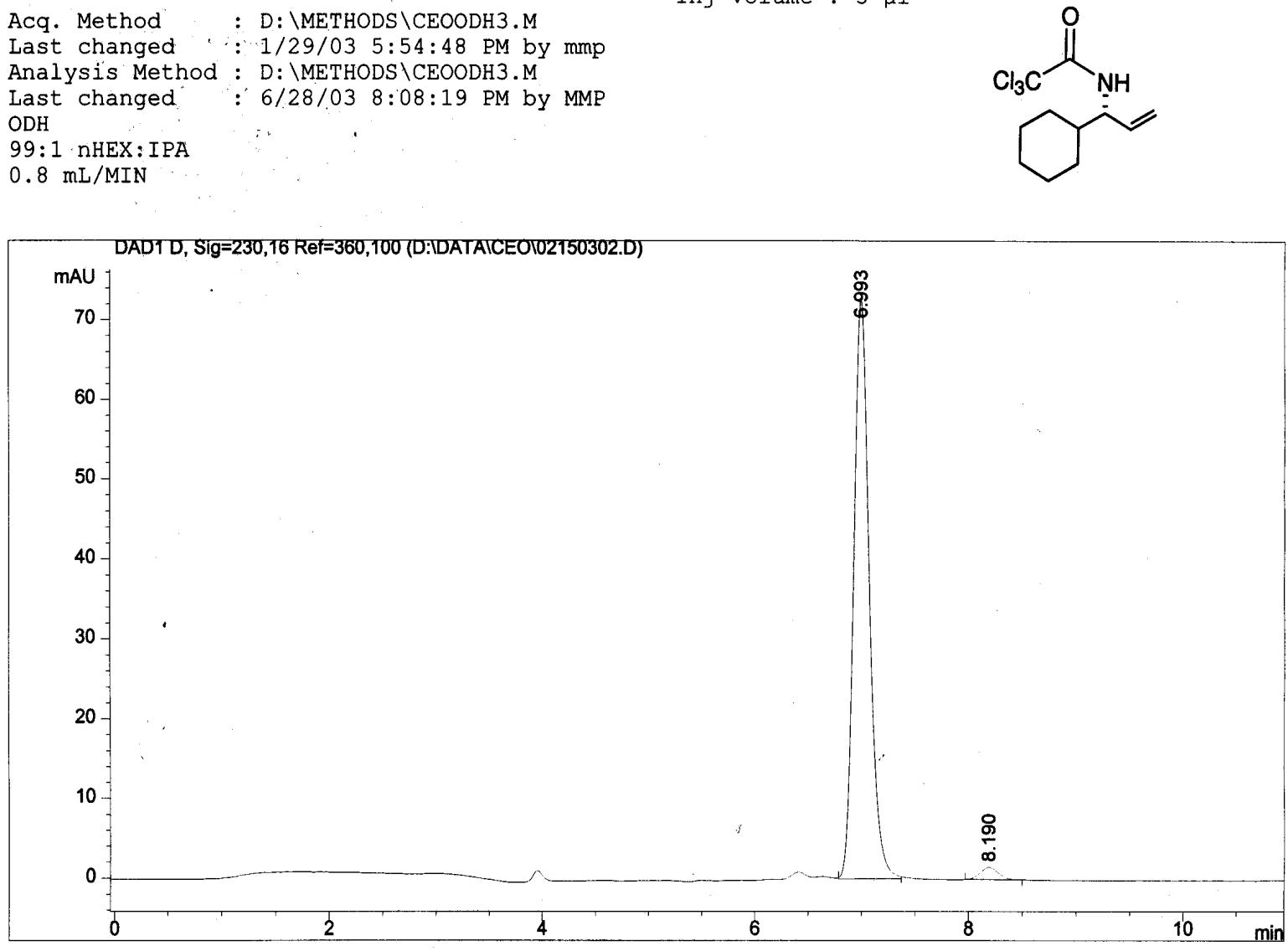

Area Percent Report

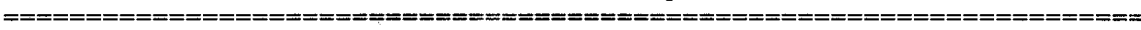

Sorted By : Signal

Multiplier $\quad: \quad 1.0000$

Dilution : $\quad 1.0000$

Use Multiplier \& Dilution Factor with ISTDS

Signal 1: DAD1 D, Sig=230,16 Ref $=360,100$

\begin{tabular}{|c|c|c|c|c|c|c|}
\hline $\begin{array}{c}\text { Peak } \\
\#\end{array}$ & $\begin{array}{c}\text { RetTime } \\
\text { [min] }\end{array}$ & Type & $\begin{array}{c}\text { Width } \\
\text { [min] }\end{array}$ & $\begin{array}{c}\text { Area } \\
{\left[\mathrm{mAU}^{*} \mathrm{~s}\right]}\end{array}$ & $\begin{array}{l}\text { Height } \\
\text { [mAU] }\end{array}$ & $\begin{array}{c}\text { Area } \\
\frac{8}{8}\end{array}$ \\
\hline $\begin{array}{l}1 \\
2\end{array}$ & $\begin{array}{l}6.993^{\circ} \\
8.190\end{array}$ & $\begin{array}{l}\mathrm{BB} \\
\mathrm{BB}\end{array}$ & $\begin{array}{l}0.1532 \\
0.1875\end{array}$ & $\begin{array}{r}731.01862 \\
18.58839\end{array}$ & $\begin{array}{r}72.75700 \\
1.53310\end{array}$ & $\begin{array}{r}97.5202 \\
2.4798\end{array}$ \\
\hline Total & . & & & 749.60701 & 7.4 .29011 & \\
\hline
\end{tabular}

Results obtained with enhanced integrator!

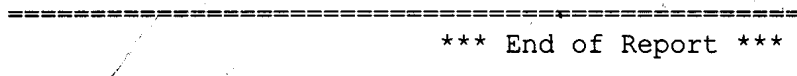


Supporting Information Anderson and Overman

Injection Date : 1/15/03 3:59:44 PM

Sample Name : ceo-V-197B

Acq. Operator : ceo

Acq. Method : D: \METHODS \CEOODH2.M

Last changed : 1/15/03 3:59:17 PM by ceo (modified after loading)

Analysis Method : D: \METHODS \CEOODH3.M

Last changed : 6/28/03 8:08:19 PM by MMP

$\mathrm{ODH}$

99:1 nHEX:IPA

$0.8 \mathrm{~mL} / \mathrm{MIN}$
Seq. Line: 11

Location : Vial 69

Inj : 1

Inj Volume : $5 \mu \mathrm{l}$

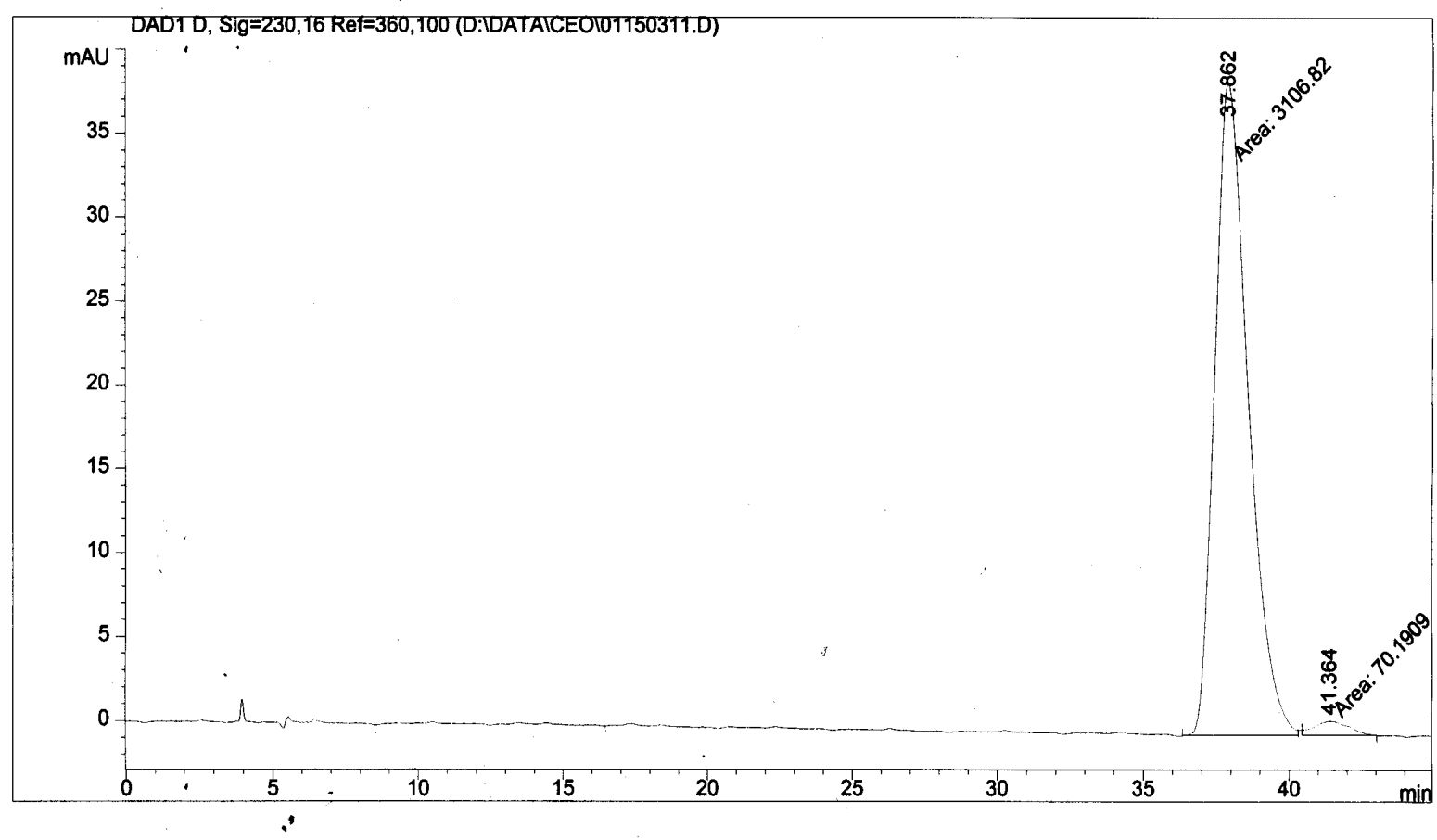




Supporting Information Anderson and Overman

Data File D: \DATA \CEO \02270307.D
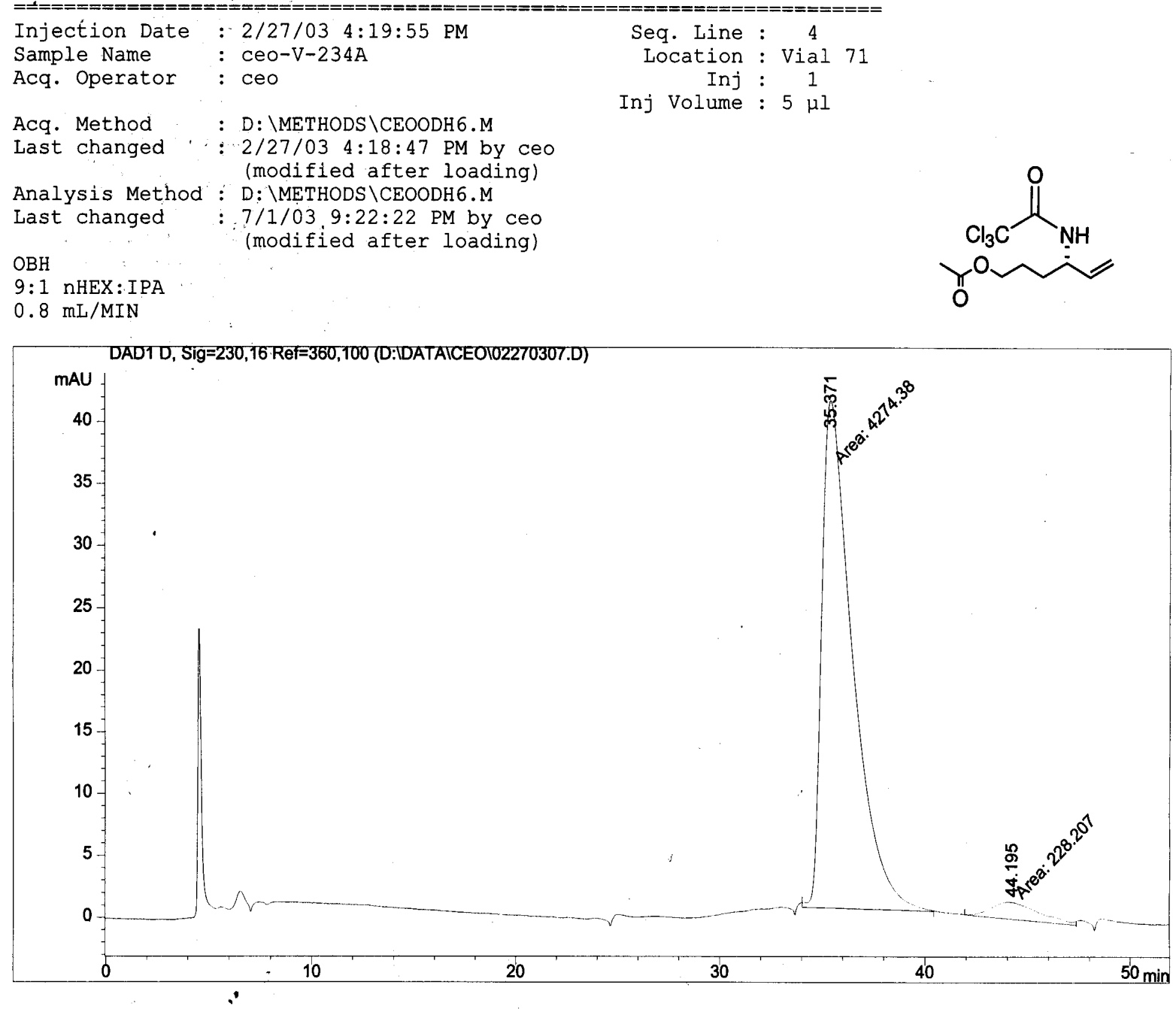

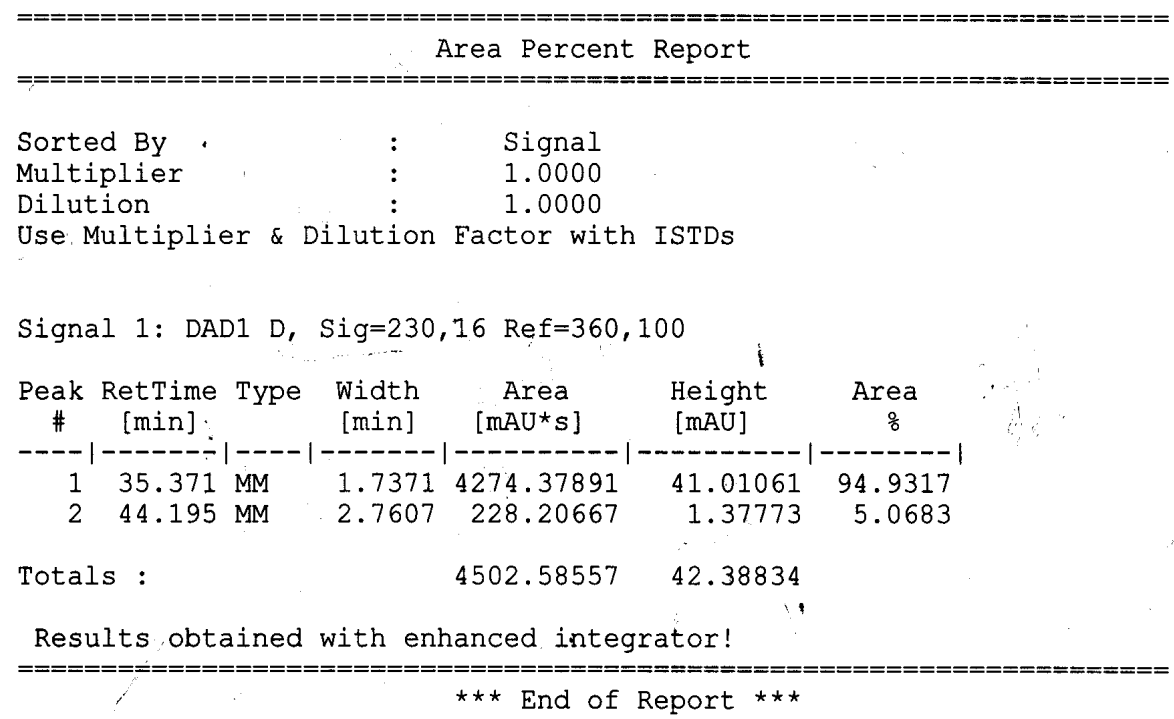


Supporting Information Anderson and Overman


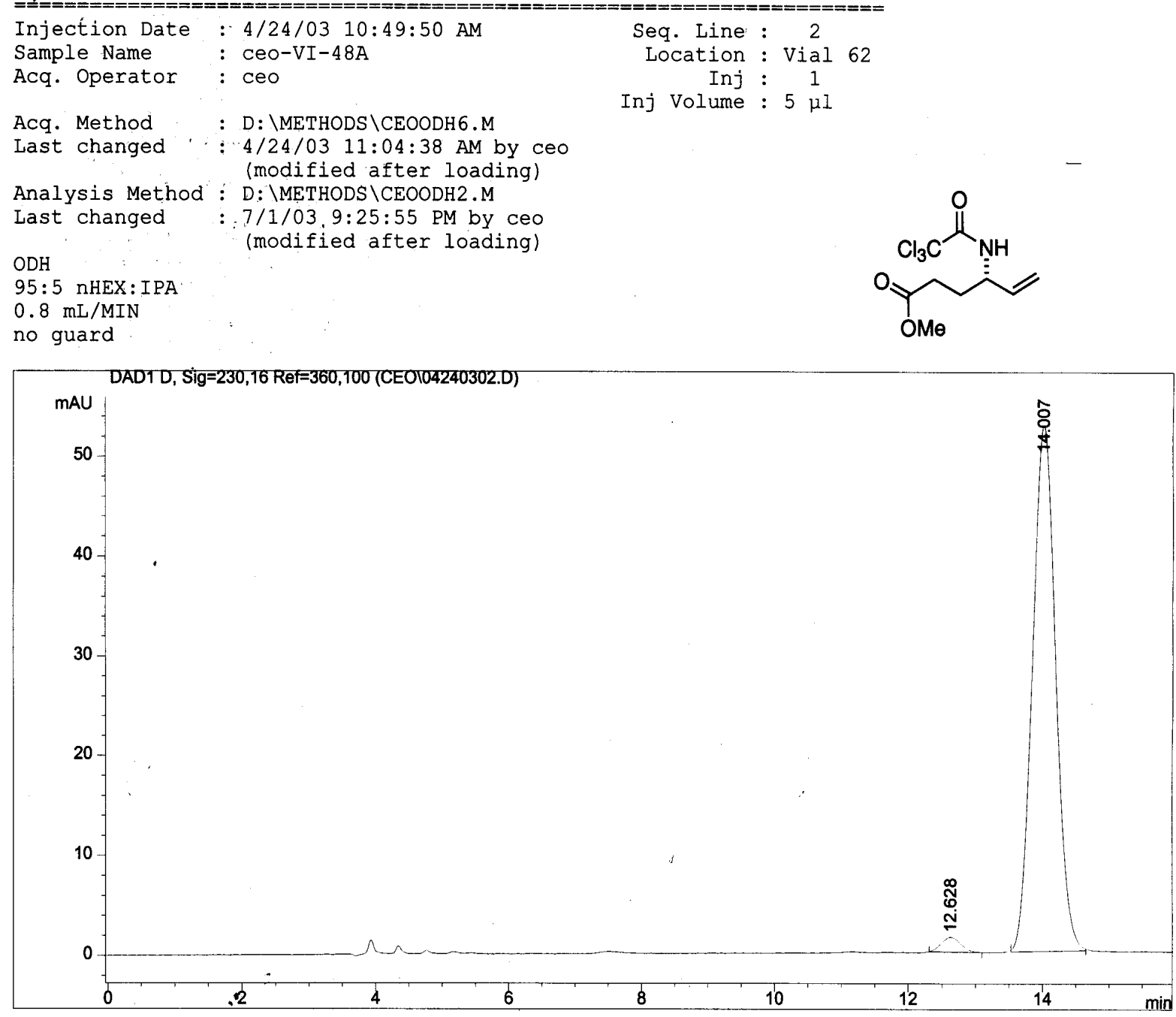

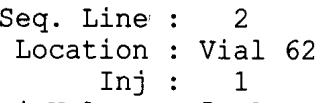

Inj Volume : $5 \mathrm{\mu l}$

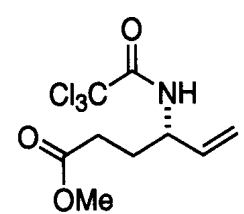

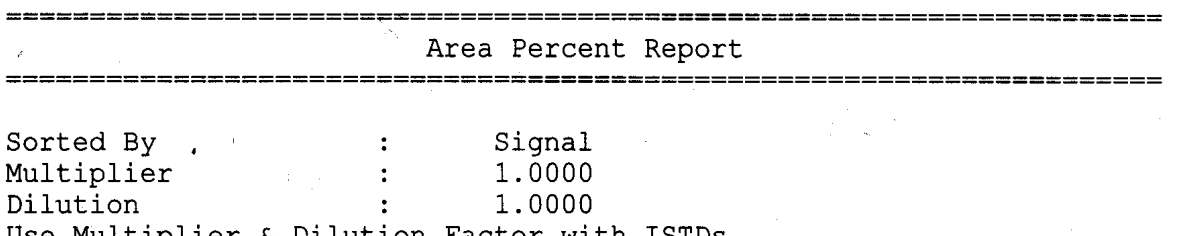

Use Multiplier \& Dilution Factor with ISTDs

Signal 1: DAD1 D, Sig=230,16 Réf $=360,100$

\begin{tabular}{|c|c|c|c|c|c|c|}
\hline $\begin{array}{c}\text { Peak } \\
\#\end{array}$ & $\begin{array}{c}\text { RetTime } \\
\text { [min] }\end{array}$ & Type & $\begin{array}{l}\text { Width } \\
\text { [min] }\end{array}$ & $\begin{array}{c}\text { Area } \\
{\left[\mathrm{mAU}^{*} \mathrm{~s}\right]}\end{array}$ & $\begin{array}{l}\text { Height } \\
{[\mathrm{mAU}]}\end{array}$ & $\begin{array}{c}\text { Area } \\
8\end{array}$ \\
\hline $\begin{array}{l}1 \\
2\end{array}$ & 12.628 & $\begin{array}{l}\mathrm{BP} \\
\mathrm{BB}\end{array}$ & $\begin{array}{l}0.2842 \\
0.3749\end{array}$ & $\begin{array}{r}29.76179 \\
1270.70056\end{array}$ & $\begin{array}{r}1.47016 \\
52.76957\end{array}$ & $\begin{array}{c}2.2886 \\
97.7114\end{array}$ \\
\hline Total & Ls: & & & 1300.46235 & 54.23974 & \\
\hline
\end{tabular}

$\star \star \star$ End of Report $* \star \star$ 
Supporting Information Anderson and Overman
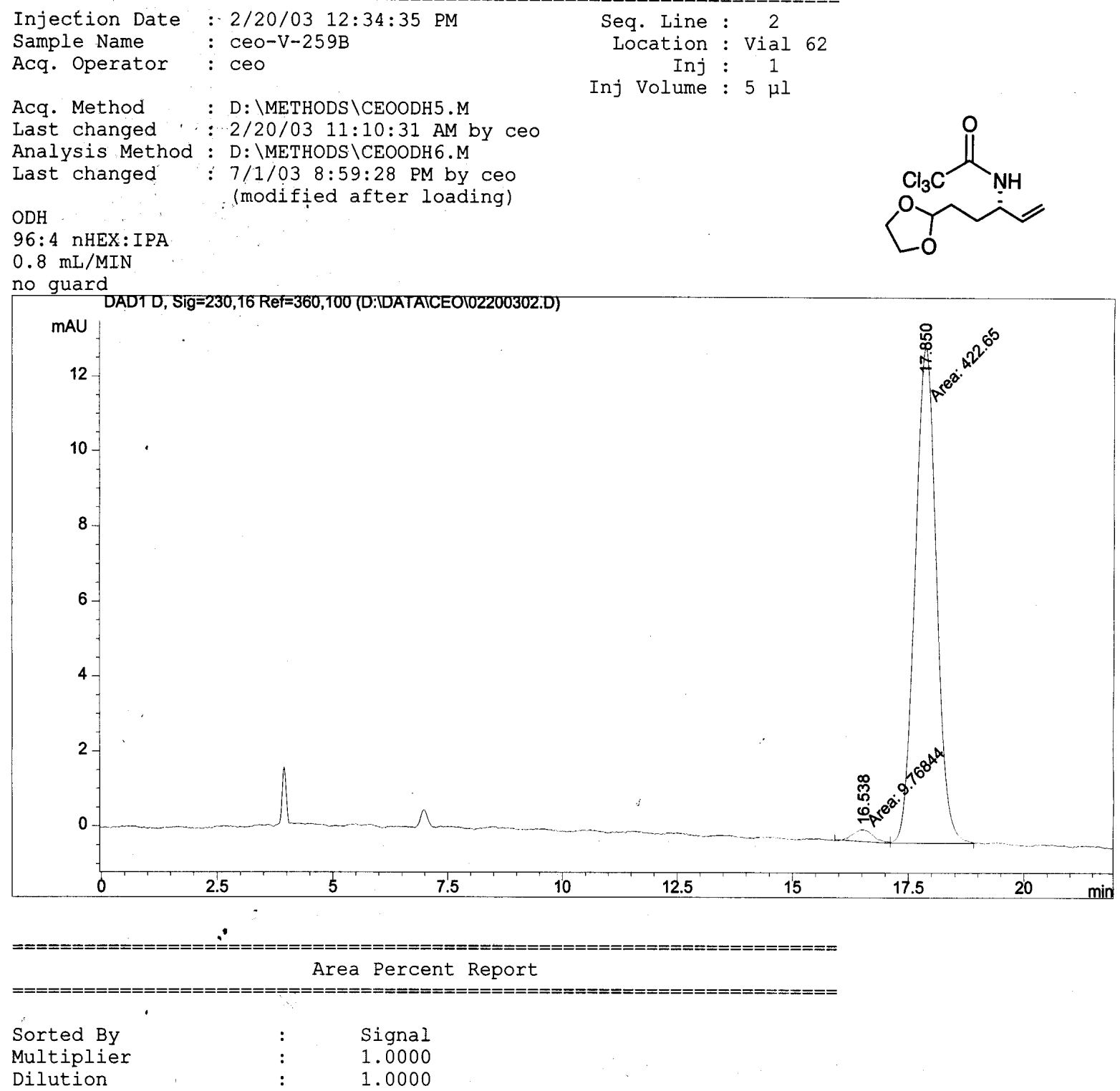

Use Multiplier \& Dilution Eactor with ISTDs

Signal 1: DAD1 D, Sig=230,16 Ref $=360,100$

\begin{tabular}{|c|c|c|c|c|c|c|}
\hline $\begin{array}{c}\text { Peak } \\
\#\end{array}$ & $\begin{array}{l}\text { RetTime } \\
\text { [min] }\end{array}$ & Type & $\begin{array}{l}\text { Width } \\
\text { [min] }\end{array}$ & $\begin{array}{c}\text { Area } \\
{\left[\mathrm{mAU}^{\star} \mathrm{s}\right]}\end{array}$ & $\begin{array}{l}\text { Height } \\
{[\mathrm{MAU}]}\end{array}$ & $\begin{array}{c}\text { Area } \\
\frac{8}{0}\end{array}$ \\
\hline $\begin{array}{l}1 \\
2\end{array}$ & $\begin{array}{l}16.538 \\
17.850\end{array}$ & $\begin{array}{l}\text { MM } \\
\text { MM }\end{array}$ & $\begin{array}{l}0.5198 \\
0.5322\end{array}$ & $\begin{array}{r}9.76844 \\
422.64951\end{array}$ & $\begin{array}{r}3.13214 \mathrm{e}-1 \\
13.23678\end{array}$ & $\begin{array}{r}2.2590 \\
97.7410\end{array}$ \\
\hline Tot & : & & & 432.41795 & 1.3 .54999 & \\
\hline
\end{tabular}

Results obtained with enhanced integrator!






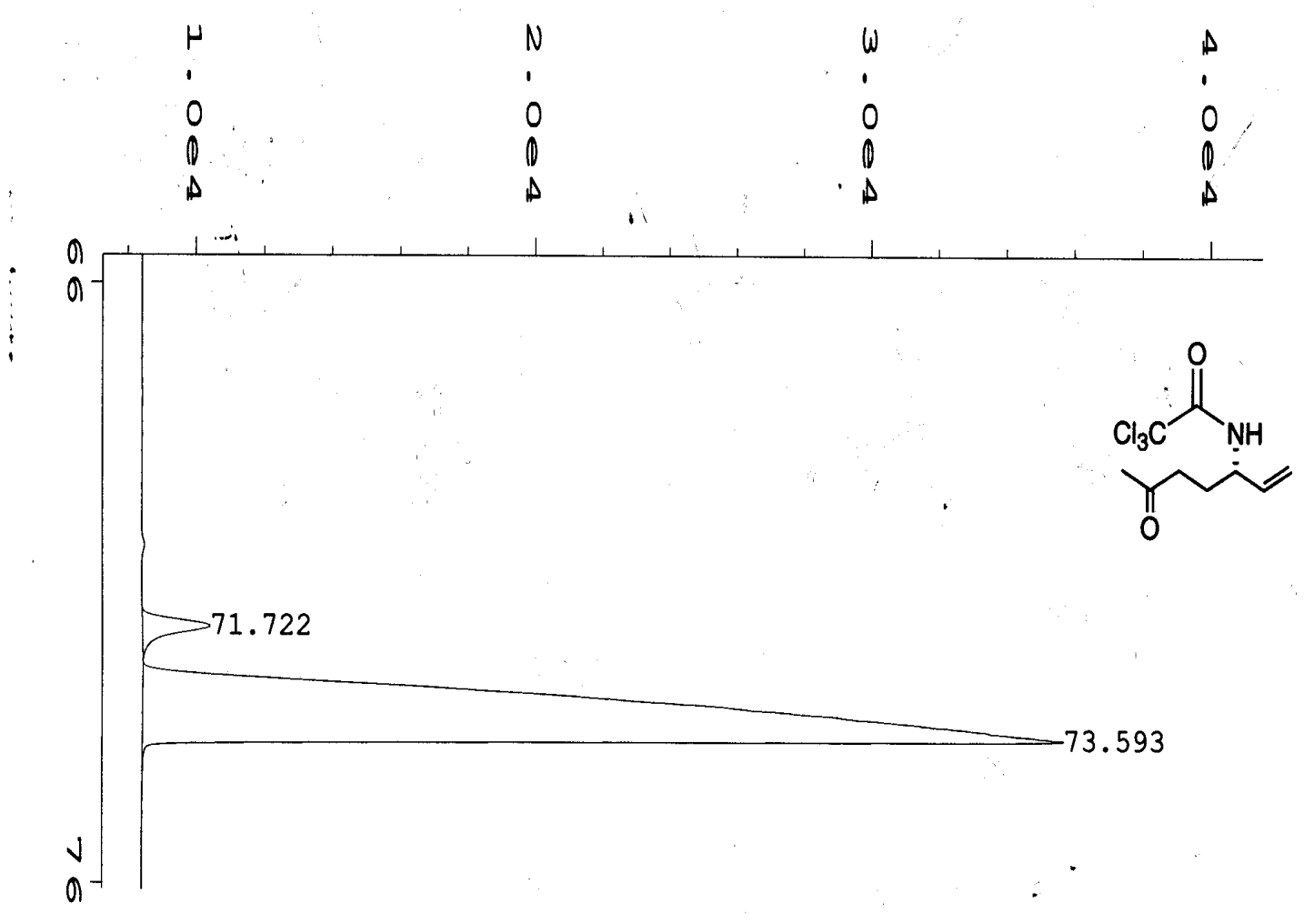

\begin{tabular}{|c|c|c|}
\hline Data File Name &  & 06-25A.D \\
\hline Operator & : ANDERSON & Page Number \\
\hline Instrument & : INSTRUMEN & Vial Number \\
\hline Sample Name & & Injection Number : \\
\hline Run Time Bar Code: & & Sequence Line : \\
\hline Acquired on & : $02 \mathrm{Jul} \quad 03 \quad 10: 30 \mathrm{AM}$ & Instrument Method: LONGRUN.MTH \\
\hline Report Created on: & : $02 \mathrm{Jul} 03 \quad 11: 47 \mathrm{AM}$ & Analysis Method : LONGRUN.MTH \\
\hline
\end{tabular}

Sig. 2 in $C: \backslash H P C H E M \backslash 2 \backslash D A T A \backslash A N D E R S O N \backslash C E 06-25 A . D$

\begin{tabular}{|c|c|c|c|c|c|c|}
\hline Pk\# & Ret Time & Area & Height & Type & Width & Area \% \\
\hline 1 & 71.722 & 32729 & 1982 & BB & 0.219 & 28187 \\
\hline 2 & 73.593 & 1128411 & 27220 & BB & 0.500 & 97.1813 \\
\hline
\end{tabular}

Total area $=1161141$ 
Supporting Information Anderson and Overman





Last changed : $: 3 / 13 / 03 \quad 12: 22: 17$ PM by ceo (modified after loading)

Analysis Method : D: \METHODS \CEOODH3.M

Last changed : 6/28/03, 8:08:19 PM by MMP

$\mathrm{ODH}$

99:1 NHEX:IPA

$0.8 \mathrm{~mL} / \mathrm{MIN}$


Area Percent Report



Use Multiplier \& Dilution Factor with ISTDs

Signal 1: DAD1 D, Sig=230, 1'6 Ref=360,100

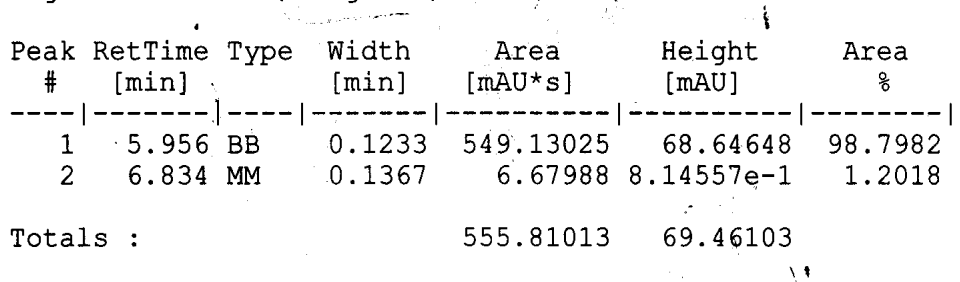

Results obtained with enhanced integrator!

$\star \star \star$ End of Report $\star * \star$ 
Supporting Information Anderson and Overman


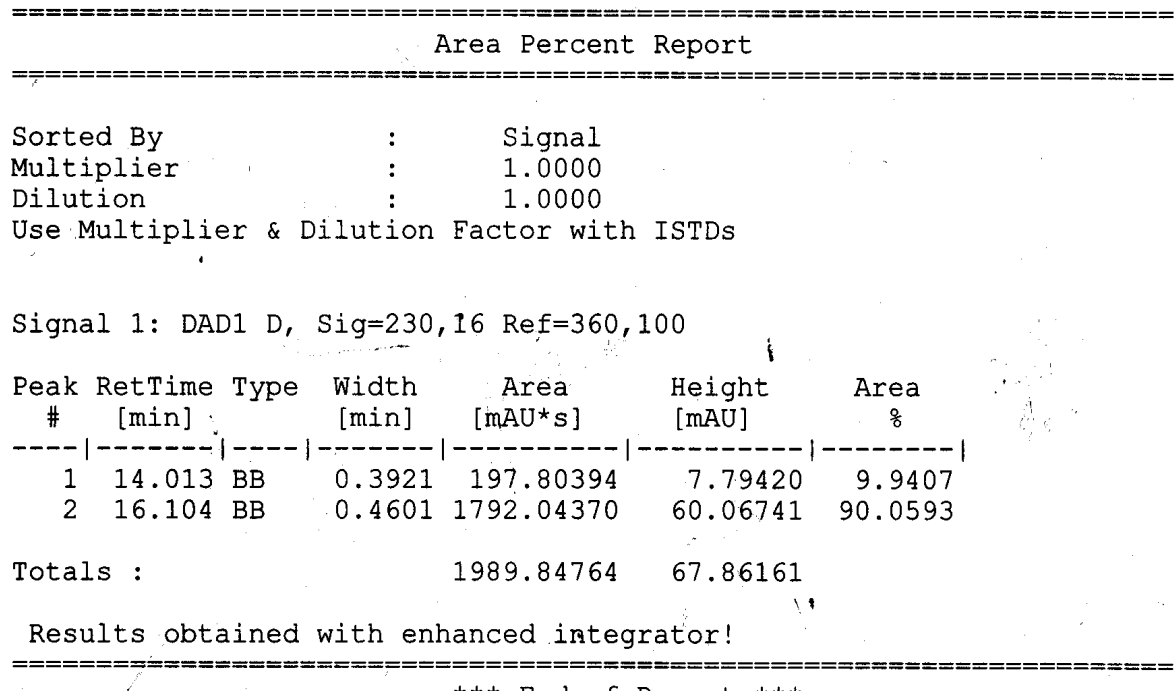


Supporting Information Anderson and Overman

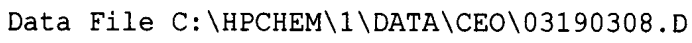
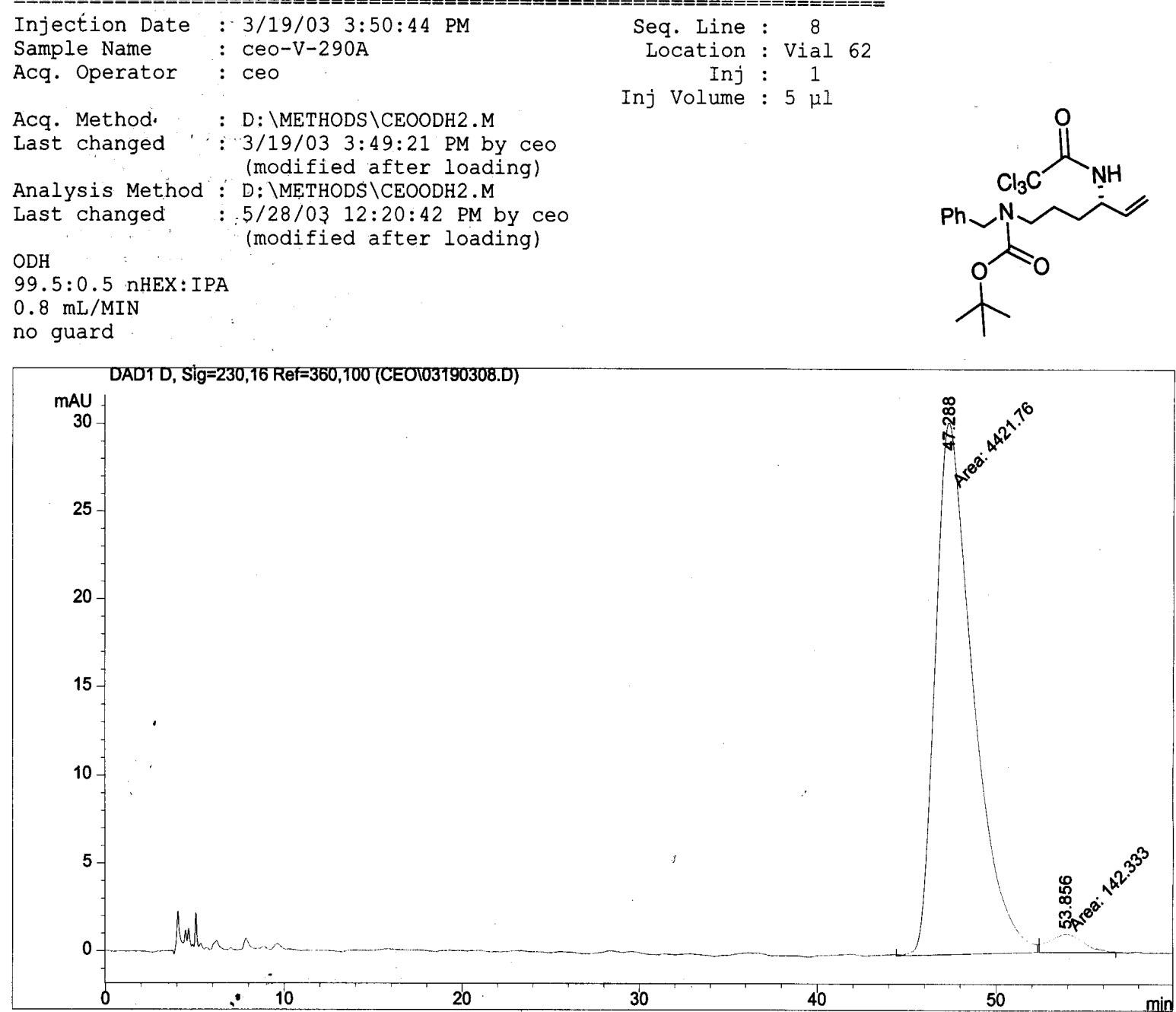

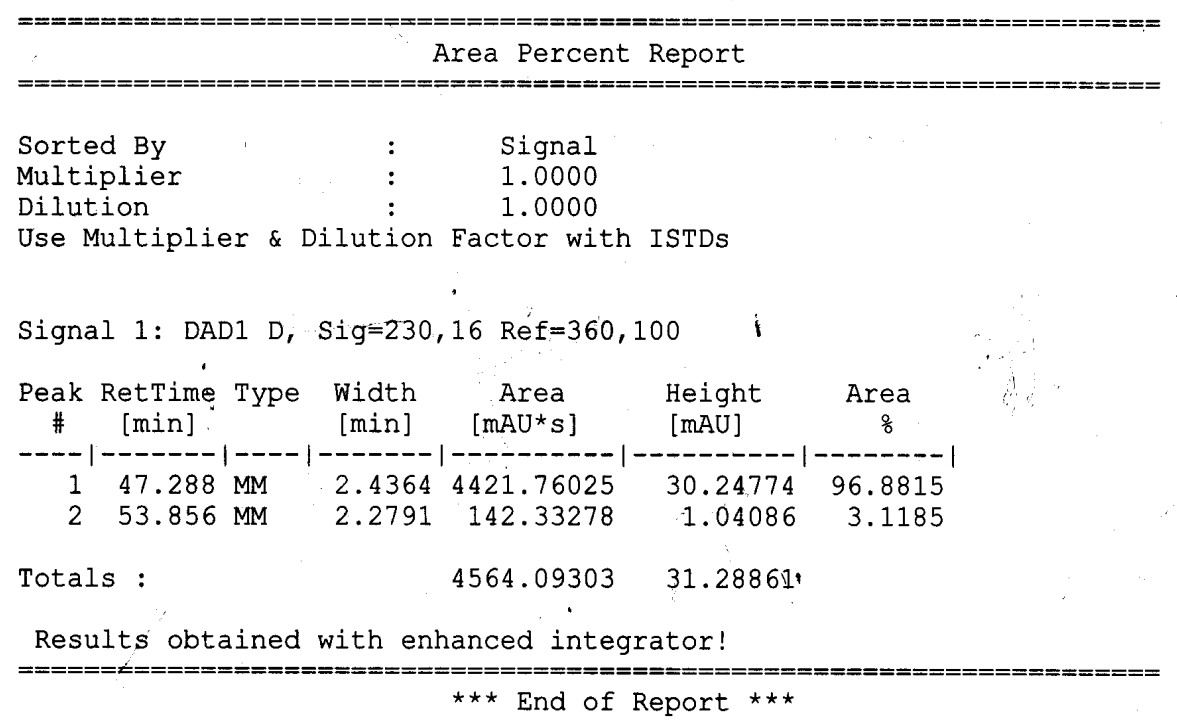


Supporting Information Anderson and Overman

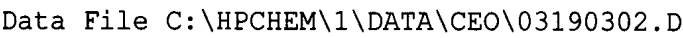
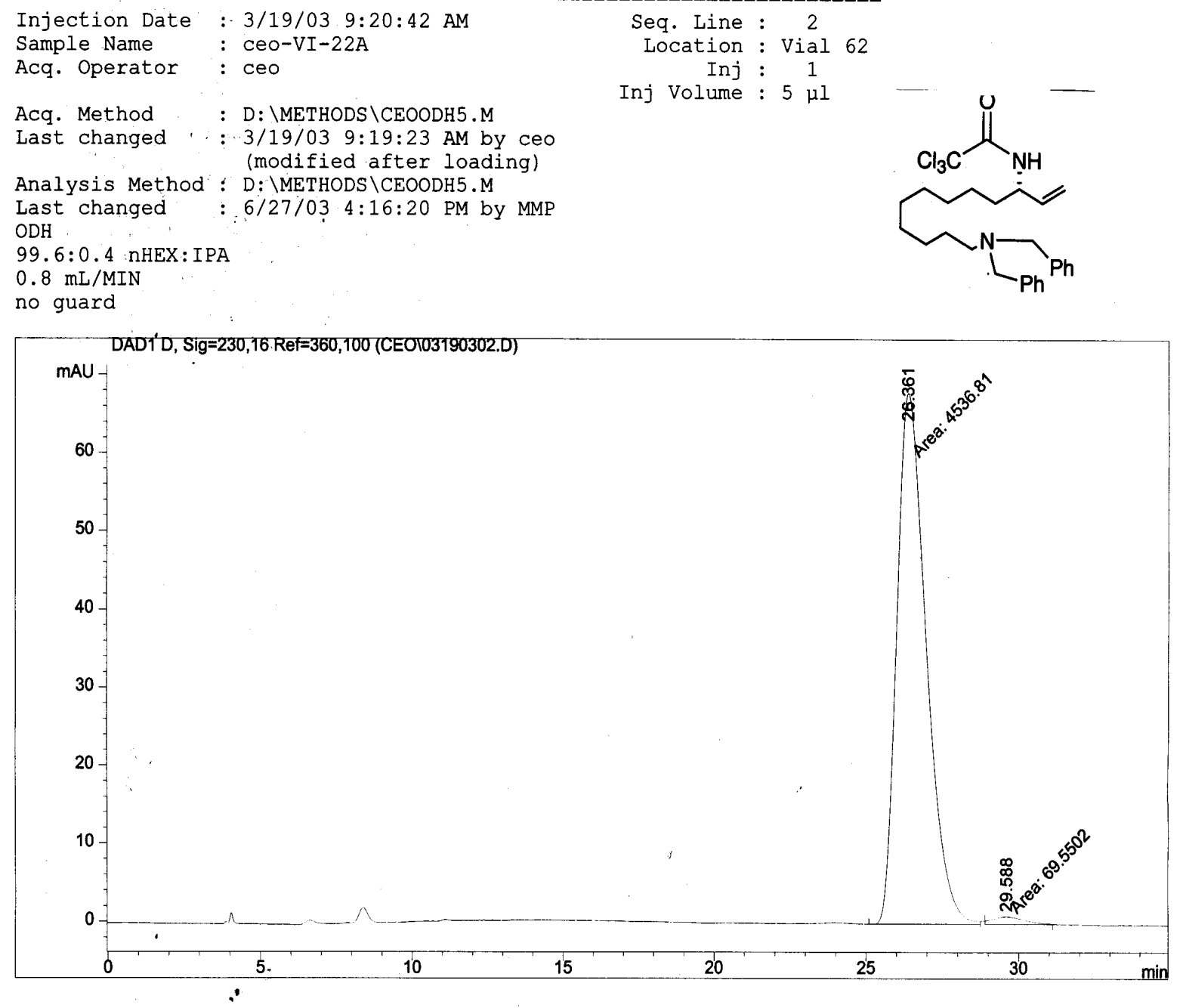

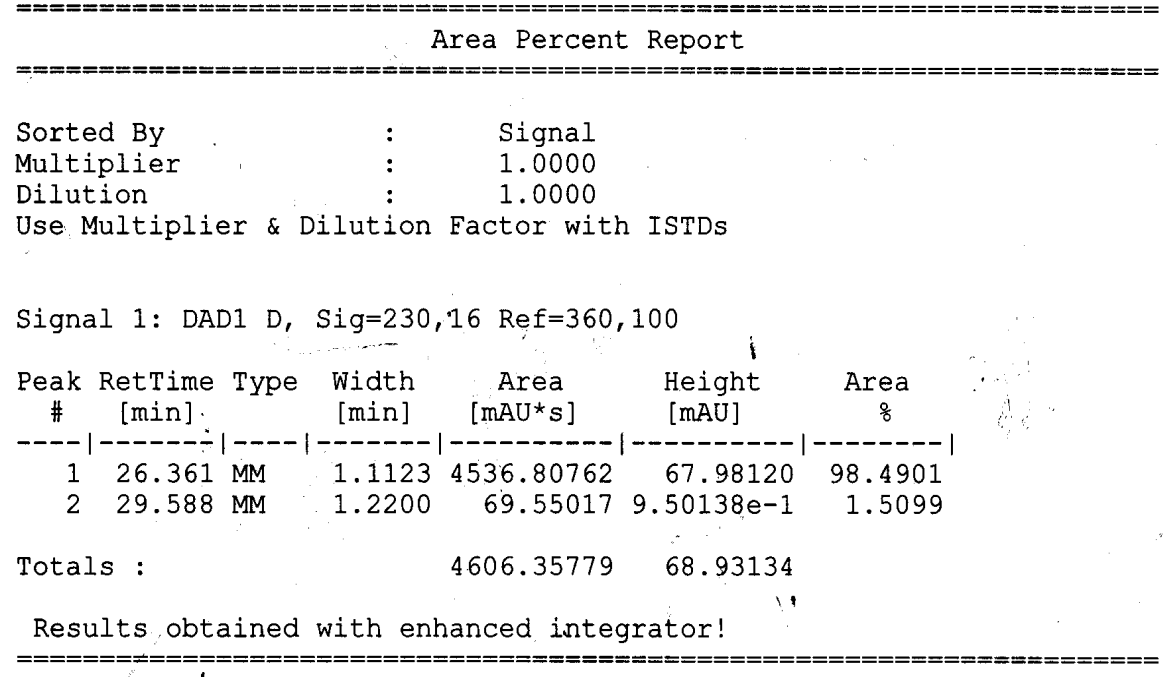

$\star \star \star$ End of Report $* \star *$ 